\title{
Algorithm portfolio based scheme for dynamic optimization problems
}

\author{
Jenny Fajardo Calderín ${ }^{1}$, Antonio D. Masegosa ${ }^{2,3}$, David A. Pelta ${ }^{4}$ \\ ${ }^{1}$ Dept. of Artificial Intelligence and Infrastructure and Systems, Polytech. Higher Inst. José A. Echeverría, \\ La Habana, Cuba \\ E-mail: jfajardo@ceis.cujae.edu.cu \\ ${ }^{2}$ Deusto Institute of Technology, University of Deusto, \\ Bilbao, Spain \\ E-mail:ad.masegosa@deusto.es \\ ${ }^{3}$ IKERBASQUE, Basque Foundation for Science, \\ Bilbao, Spain \\ ${ }^{4}$ Dept. of Computer Science and AI, Research Center for ICT (CITIC-UGR), University of Granada, \\ Granada, Spain \\ E-mail:dpelta@decsai.ugr.es
}

Received 18 December 2014

Accepted 4 March 2015

\begin{abstract}
Since their first appearance in 1997 in the prestigious journal Science, algorithm portfolios have become a popular approach to solve static problems. Nevertheless and despite that success, they have not received much attention in Dynamic Optimization Problems (DOPs). In this work, we aim at showing these methods as a powerful tool to solve combinatorial DOPs. To this end, we propose a new algorithm portfolio for this type of problems that incorporates a learning scheme to select, among the metaheuristics that compose it, the most appropriate solver or solvers for each problem, configuration and search stage. This method was tested over 5 binary-coded problems (dynamic variants of OneMax, Plateau, RoyalRoad, Deceptive and Knapsack) and compared versus two reference algorithms for these problems (Adaptive Hill Climbing Memetic Algorithm and Self Organized Random Immigrants Genetic Algorithm). The results showed the importance of a good design of the learning scheme, the superiority of the algorithm portfolio against the isolated version of the metaheuristics that integrate it, and the competitiveness of its performance versus the reference algorithms.
\end{abstract}

Keywords: algorithm portfolio, dynamic optimization problems, learning, algorithm selection problem, combinatorial problems

\footnotetext{
*This work has been supported by the research projects TIN2011-27696-C02-01 and TEC2013-45585-C2-2-R from the Spanish Ministry of Economy and Competitiveness, P11-TIC-8001 from the Andalusian Government (including FEDER funds from the European Union), and PC2013-71A from the Basque Government. Jenny Fajardo has also been supported by a scholarship from the Ibero-American University Association for Post Graduate Studies (AUIP).
} 


\section{Introduction}

An optimization problem can be considered as "dynamic" when any of its components, namely objective function, constraints, size, variables domain, etc., change with time. Dynamic optimization problems (DOPs) are as follows:

$$
\text { DOPs }=\{\text { optimize } f(x, t) ; x \in F(t) \subset S ; t \in T\}
$$

where:

- $S$ is the search space

- $t$ represents time

- $f: S \times T \longrightarrow R$ is the objective or cost function whose definition depends on $t$.

- $F(t)$, is the set of feasible solutions at time $t$, $F(t) \subset S$.

Most of the academic research in DOPs has been done using artificial problems, because they allow to properly studying several factors related with the changes, as severity, frequency or dynamism type. Examples of artificial problems are the wellknown moving peaks benchmark [28, 29]; dynamic versions of static continuous functions like Sphere, Griewank, Rastrigin, Ackley, etc. [23]; or dynamic versions of combinatorial problems as knapsack problem [3, 21, 35], moving parabola [1], bitwise comparison problems $[10,41]$, and planning problems $[8,11,26,37,38]$.

When dealing with DOPs, some aspects are assumed: the changes are gradual; it is not possible to solve the problem from scratch after a change; and the current information should be useful to produce a faster adaptation to the change.

The last years showed an increasing interest on solving them using metaheuristic methods $[2,7,30$, 49,51]. One can find evolutionary algorithms [50], multiswarm techniques [2], ant colony optimization $[19,27]$, cooperative strategies $[17,18,25]$ and so on.

When solving static problems with metaheuristics, parameter setting is not a trivial task. As a consequence, researchers enhance their methods with some learning features to change the parameters during the run. This is also being done in the context of DOPs, where there is a growing tendency on using learning mechanism to change the parameters, operators, etc. of the method during the search $[17,18,24,27,46,51]$.

Another non trivial aspect is the selection of the metaheuristic to solve the problem at hand. In the context of static problems, one of the most common approaches for this issue is the algorithm portfolio [20,31,32]. It consists in a set of algorithms that are executed iteratively or in parallel, in order to solve the problem. A learning scheme is used to select the most suitable algorithm at every stage of the search or to distribute the available execution time among them. This decision is typically based on the algorithm's performance. This type of methods have shown to be very competitive in problems as supply chain optimization [48], numerical optimization [32], vehicle routing [39] or satisfiability [47], among others.

Despite this success in static problems, algorithm portfolios have not received much attention in DOPs. For this reason, in this paper we intend to deepen in this topic, to show algorithm portfolios as a powerful alternative to solve DOPs. Concretely, we propose a new algorithm portfolio for combinatorial DOPs emphasizing the role of the learning scheme.

First, we will analyze if learning makes sense, what learning scheme is the most appropriate and how the learning works along the search process. Then we will study if the portfolio obtains better results than their composing algorithms, and finally, we will compare the best portfolio variant against two reference algorithms: Adaptive Hill Climbing Memetic Algorithm and Self Organized Random Immigrants Genetic Algorithm. The computational experiments are done over five combinatorial optimization problems and the results are analyzed using statistical testing. It is important to remark that we will consider DOPs with dynamism only in the fitness function.

The article is organized as follows. Section 2 discusses the literature related to our proposal. The algorithm portfolio, its learning scheme and the different learning variants considered are presented in Section 3. In Section 4, experimental framework, we show the combinatorial DOPs used to test the 
method, the details of the experimentation, the reference algorithms with which our proposal is compared, the composition of the portfolio, the performance measures and the non-parametric tests employed for the statistical assessment of the results. The next part of the paper is devoted to analyze the results of the experimentation and the comparison versus the reference algorithms. Finally, we provide the conclusions of this work in Section 6.

\section{Related Work}

The idea of algorithm portfolios was proposed by Huberman and coauthors in 1997 [20]. Inspired by basic economic concepts of risk management, these authors presented an approach to allocate a limited amount of resources (CPU time) among a set of algorithms according to its expected "benefit" (average fitness) and "risk" (fitness variance). The allocation is done in such a way that the benefit is maximized and the risk minimized.

The original concept has evolved along these years and nowadays, one of the most accepted definitions is the next one [16]: a collection of different algorithms and/or copies of the same algorithm that are run in one or more CPUs. We can classify algorithm portfolios in different categories depending on: 1) how the algorithms are run and 2) when the available running time is distributed among them $[16,33]$. Regarding the first criterion, there are three classes [16]: parallel, where all the algorithms are run concurrently in different processors [32]; interleaved on a single processor, where the algorithms are run alternatively, simulating parallelism in one processor [14]; and sequential with restart, where at each iteration, a randomly selected algorithm is executed for a fixed amount of time (every run of the same algorithm uses a different random seed) [14]. Respect to the second criterion, the algorithm portfolios can be classified in two categories [14,33]: static, if the distribution of the available CPU time among the algorithms is fixed before the run [32,48], or dynamic, if it is done along the search process [14].

We can find in the literature some references about the application of algorithm portfolios to solve
DOPs. For example, in [40], the authors propose a static portfolio to solve the Inventory Routing Problem with stochastic demands. In this case, the algorithms are run in parallel without information feedback, and the portfolio is composed by variants of the genetic algorithm. Another work on this topic is found in [39], where an algorithm portfolio is used to solve the Dynamic Vehicle Routing Problem with stochastic demands. The paper presents a static algorithm portfolio that combines trajectorybased and population-based algorithms that are run in parallel with no information exchange.

A similar approach to algorithm portfolios that has been used in DOPs are the hyperheuristics [5]. They can be defined as search methods or learning mechanisms for the selection and generation of heuristics in order to solve a particular optimization problem. They can also be seen as high-level methods that given a set of low-levels heuristics for a particular problem, are able to automatically produce a proper combination of these low-level heuristics to solve the instance at hand. Among the high-level methods for the selection/generation/combination of heuristics we can find tabu search, variable neighborhood search, genetic algorithms or data mining techniques [5]. In the context of static problems, these methods have been applied to graph coloring, production planning, work-force scheduling, constraint satisfaction or vehicle routing [5,6].

Regarding DOPs, its application is fairly recent. In [45], Gonul et al. proposed a framework for DOPs that hybridizes hyperheuristics and Population Based Incremental Learning. The selection of low-level heuristics is done through a scoring system and reinforcement learning. They tested two variants of their proposal on dynamic binary functions with successful results. Topcuoglu and coauthors present a hyperheuristic to solve DOPs in [43] whose high-level heuristic selection method is an evolutionary technique known as memory/search algorithm. The experimentation done over the Dynamic Generalized Assignment Problem and the Moving Peaks Benchmark showed the better performance of this method versus the canonical memory/search algorithm. In [22], the authors test, using also the Moving Peaks Benchmark, several hy- 
perheuristics that combine five high-level heuristic selection methods (simple random, greedy, choice function, reinforcement learning, random permutation descent) with seven move acceptance criteria (all moves, only improving, improving and equal, exponential Monte Carlo with counter, great deluge, simulated annealing, simulated annealing with reheating). The best performing variant (choice function - improving and equal) improved the results obtained by state-of-the-art algorithms for this problem.

As we have seen, the use of algorithm portfolios and hyperheuristics is becoming more popular in DOPs. In this sense, although algorithm portfolios and hyperheuristics share a similar approach (they combine a set of heuristics), it is important to highlight that they present two important differences. On one hand, hyperheuristics usually work with low-level heuristics whereas algorithm portfolios do it with higher-level methods as metaheuristics. On the other hand, and in our opinion the main difference, hyperheuristics works over a single solution of the problem (they can be seen as trajectorybased methods) while algorithm portfolios works over a set of independent solutions at the same time. Taking into account these two aspects, the characteristics of our proposal, which we describe in the next section, fits better to the category of algorithm portfolios, since our method deals with metaheuristics that works over their own and independent solutions.

\section{Algorithm Portfolio}

The main motivation to build an algorithm portfolio is to avoid deciding on a single algorithm to solve the problem at hand [20]. In this contribution, our portfolio is made of a set of metaheuristics. Using a credit based approach, the portfolio selects which metaheuristic to run at every stage of the search. Then, a credit assignment is done based on the performance of the selected metaheuristic. According to the categories of algorithm portfolios showed in the last section, our proposal can be classified as a dynamic algorithm portfolio that interleaves the run of the metaheuristics on a single processor.

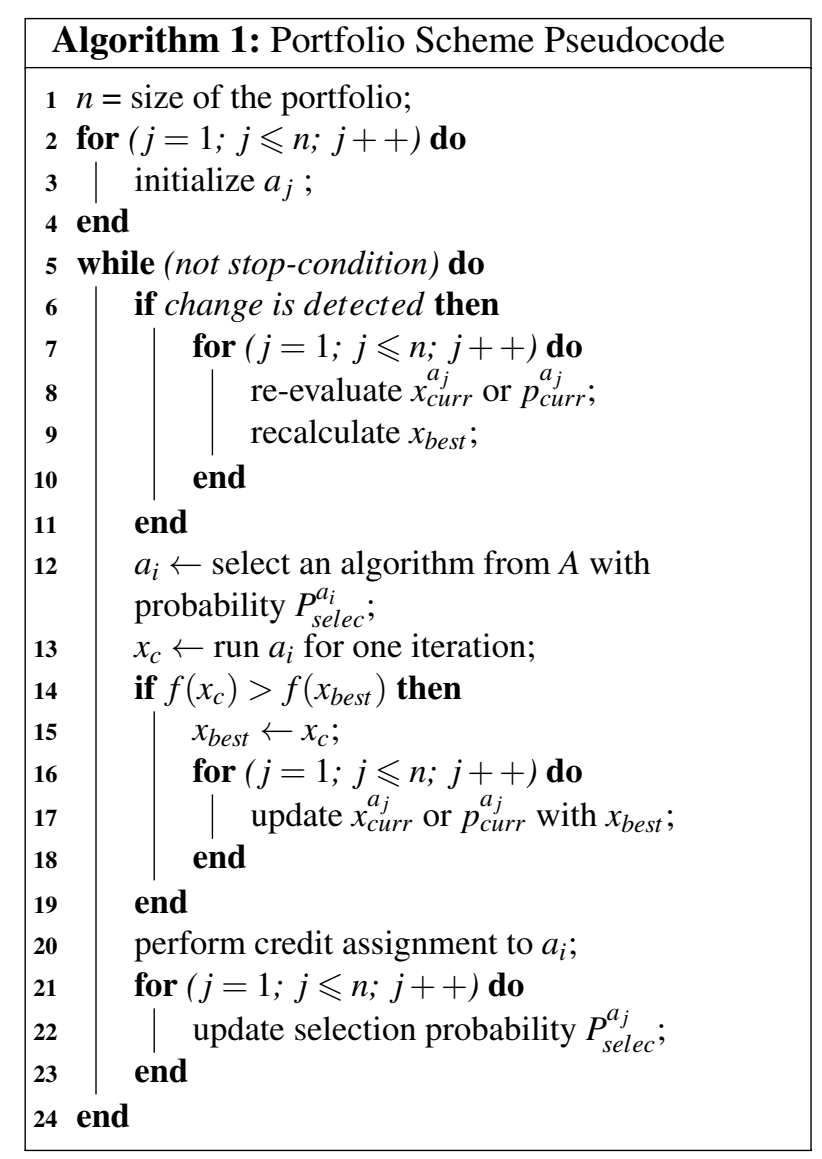

More formally, our portfolio is composed by a set of metaheuristics $\mathrm{A}=\left\{a_{1}, \ldots, a_{n}\right\}$, being each $a_{i}$ a trajectory or population-based metaheuristic. Every $a_{i}$ has a single current solution $x_{\text {curr }}^{a_{i}}$ (if we consider a trajectory-based method) or a current population $p_{c u r r}^{a_{i}}$ (in population-based metaheuristics).

Algorithm 1 shows the inner working of the portfolio. After the initialization of the algorithms, the method goes into the main loop. Firstly, if a change is detected, $x_{\text {curr }}^{a_{i}}$ or $p_{\text {curr }}^{a_{i}}$ are re-evaluated, and the new global best solution $\left(x_{\text {best }}\right)$ is calculated.

Subsequently, an $a_{i}$ is selected and run for one iteration (the definition of what we consider by an iteration is given at the end of this section). The selection strategy employed in this step is the wellknown roulette-wheel method, where the portion or selection probability of each $a_{i}$ is assigned according to its credit $w_{i}$. This probability is calculated as:

$$
P_{\text {selec }}^{a_{i}}=\frac{w_{i}}{\sum_{j=1}^{n} w_{j}}
$$


Table 1. Details of the steps the algorithm portfolio accomplishes in a different way depending on whether the selected algorithm is trajectory-based or population-based.

\begin{tabular}{lll}
\hline Step (Pseudocode line in Algorithm 1) & Trajectory-based & Population-based \\
\hline Algorithm initialization (3) & $\begin{array}{l}\text { random generation of a single solution } \\
\text { following a uniform distribution }\end{array}$ & $\begin{array}{l}\text { random generation of the population } \\
\text { following a uniform distribution }\end{array}$ \\
\hline Re-evaluation (8) & re-evaluate $x_{\text {curr }}^{a_{i}}$ & reevaluate the whole population $p_{\text {curr }}^{a_{i}}$ \\
\hline One iteration (13) & $\begin{array}{l}\text { application of the neighborhood opera- } \\
\text { tor to } x_{\text {curr }}^{a_{i}} \text { and evaluation of the accep- } \\
\text { tance criterion for the move }\end{array}$ & $\begin{array}{l}\text { perform a sequence of applications of } \\
\text { the corresponding operators }\end{array}$ \\
\hline$x_{c}(13)$ & $\begin{array}{l}\text { solution resulting from applying the } \\
\text { neighborhood operator to } x_{\text {curr }}^{a_{i}}\end{array}$ & $\begin{array}{l}\text { individual generated in the sequence of } \\
\text { applications of the operators }\end{array}$ \\
\hline$x_{\text {curr }}^{a_{i}} / p_{\text {curr }}^{a_{i}}$ update with $x_{\text {best }}(17)$ & replace $x_{\text {curr }}^{a_{i}}$ by $x_{\text {best }}$ & $\begin{array}{l}x_{\text {best }} \text { replace the worst individual in the } \\
\text { population } p_{c u r r}^{a_{i}}\end{array}$ \\
\hline
\end{tabular}

The solution generated by the algorithm selected in one iteration, $x_{c}$, is then compared versus the current global best $x_{\text {best }}$. If $x_{c}$ is better than $x_{\text {best }}$, the algorithm portfolio refreshes it and then, it updates the current solution or population of the rest of algorithms $\left(a_{j} \neq a_{i}\right)$ with the new $x_{\text {best }}$ (the details of the obtaining of $x_{c}$ and the updates of the current solutions and populations are also described at the end of the section).

In the last stage of the main loop, the method assigns a certain credit to $a_{i}$ and recalculates the selections probabilities of the algorithms according to Equation 1. The amount of credit assigned to $a_{i}$ depends on the quality of the solution $x_{c}$ and the credit scheme employed. The details of this part of the algorithm portfolio will be described in the next subsection.

Some of the steps mentioned above are accomplished in a different way depending on whether the considered algorithm is trajectory-based or population-based. Table 1 shows how the algorithm portfolio carries out these steps in each case. The most important differences appears in how an iteration is performed, how $x_{c}$ is obtained and how the current solution or population is updated. Regarding the iteration process and the obtaining of $x_{c}$, in trajectory-based algorithms, one iteration corresponds to one application of the neighborhood operator to $x_{\text {curr }}^{a_{i}}$ and the evaluation of the acceptance criterion for the move (e.g., tabu and aspiration criteria in Tabu Search, acceptance probability in Simulated Annealing, etc.). $x_{c}$ represents the solution resulting from the application of the neighborhood operator. In population-based methods, one iteration corresponds to the sequential application of their operators (e.g., selection $\rightarrow$ crossover $\rightarrow$ mutation $\rightarrow$ replacement, in Genetic Algorithms). When a crossover operator is applied, only one of the two individuals obtained (randomly selected according to a uniform distribution) is considered for the next step of the sequence of operator applications. In case the method considers replacement, the individual generated replaces the worst parent. $x_{c}$ corresponds to the individual generated in this process.

The update of $x_{\text {curr }}^{a_{i}}$ for trajectory-based algorithms, performed in the step 17 of Algorithm 1, consists on replacing $x_{\text {curr }}^{a_{i}}$ by $x_{\text {best }}$. Regarding population-based algorithms, in this step, $x_{\text {best }}$ replaces the worst individual of the population.

\subsection{Learning scheme}

The credit assignment mechanism implemented by the portfolio is, in fact, a learning scheme whose objective is to learn which the best performance $\mathrm{method} / \mathrm{s}$ for the problem at hand is/are. For this reason, we will also refer to the credit assignment mechanism as learning scheme.

The credit assigned to a metaheuristic $a_{i}$ at time $t+1$ is calculated as:

$$
w_{i}(t+1)=w_{i}(t)+r_{i}(t)-l_{i}(t)
$$

where:

- $t$ is the current time. 
- $w_{i}(t+1)$ is the credit obtained by $a_{i}$ at time step $t+1$.

- $w_{i}(t)$ is the current credit of $a_{i}$.

- $r_{i}(t)$ is the reward assigned to $a_{i}$. It is a value greater or equal to zero that is determined as a function of the quality of the solution generated by $a_{i}\left(x_{c}\right)$ and the quality of $x_{\text {best }}$.

- $l_{i}(t)$ is the penalization assigned to $a_{i}$ if the generated solution $\left(x_{c}\right)$ is worse than $x_{\text {best }}$. It is defined as follows:

$$
l_{i}(t)=w_{i}(t) * Q
$$

where: $Q$ is a penalty term and $Q \in[0,1]$.

We assume here that every possible definition or variant for the reward, penalization and credit update, lead to a different learning scheme.

\subsection{Learning scheme variants}

When facing dynamic optimization problems, it is critical to decide what to do with the learning gained (credit assigned) by the portfolio. The potential definitions of the three components of the learning scheme, namely $w_{i}(t), r_{i}(t)$ and $l_{i}(t)$, lead to variations that will be analyzed next in order to detect the best alternatives. The potential definitions are described next:

\section{Current credit $\left(w_{i}(t)\right)$ :}

- Restart (RS): current credit is set to zero when a change is detected. The rationale behind this idea is that we need to face a new problem and we need to detect from scratch which are the good methods for the new situations (we forget everything we learnt).

- Keep it or no-restart (NRS): we assume that the new situation is similar to the previous one and if a method was good in the past, it will be good in the future.

\section{Penalization $\left(l_{i}(t)\right)$ :}

- Active Penalization (AP): $Q=0.9$ if $a_{i}$ generates worse solutions than $x_{\text {best }}$, and $Q=0$ otherwise. In this way, we lower the credit of a method if it is not allowing the improvement of the best found solution. The value $Q=0.9$ was chosen after empirical observations.

- Inactive Penalization (IP): $Q=0$ during the whole run.

\section{$\operatorname{Reward}\left(r_{i}(t)\right)$ :}

- Better (RB): $r_{i}(t)=1$ if the fitness of the generated solution $\left(x_{c}\right)$ is strictly better than $f\left(x_{\text {best }}\right)$.

- Equal or Better (REB): $r_{i}(t)=1$ if the fitness of the generated solution $\left(x_{c}\right)$ is equal or better than $f\left(x_{\text {best }}\right)$.

We analyze the eight possible variants derived from the combination of the previous alternatives (shown in Table 2). As we stated above, every combination can be considered as a "learning scheme".

Table 2. Learning scheme variants

\begin{tabular}{lccc}
\hline Variant & $w_{i}(t)$ & $l_{i}(t)$ & $r_{i}(t)$ \\
\hline RS-AP-RB & RS & AP & RB \\
RS-AP-REB & RS & AP & REB \\
RS-IP-RB & RS & IP & RB \\
RS-IP-REB & RS & IP & REB \\
NRS-AP-RB & NRS & AP & RB \\
NRS-AP-REB & NRS & AP & REB \\
NRS-IP-RB & NRS & IP & RB \\
NRS-IP-REB & NRS & IP & REB \\
\hline
\end{tabular}

\section{Experimental Framework}

We describe here the problems, performance measure, comparison techniques and the details of the experimentation we will follow to evaluate our proposal.

\subsection{Problems}

The portfolio will be tested on dynamic versions of static binary-coded problems constructed using the XOR-DOP generator [51]. The generator operates generating masks that are applied to the solution using a bitwise XOR operator. The objective function is changed every $\tau$ evaluations of the fitness function. In the $k^{t h}$ change, a new XOR mask $M(k)$ is generated as follows: 


$$
M(k)=M(k-1) \oplus T(k)
$$

where $\oplus$ is the XOR operator $(a \oplus b=1 \Longleftrightarrow a \neq b$, and $a \oplus b=0$ otherwise) and $T(k)$ is an intermediate binary mask randomly generated with $\rho \times m$ values set to 1 at change $k$ (being $m$ the dimension of the problem). When $k=1, M(1)=\{0 \ldots 0\}$. The cost of a solution $x$ at evaluation $e$ is done as follows:

$$
f(x, e)=f(x \oplus M(k))
$$

where $k=\lceil e / \tau\rceil$ is the current change and \lceil\rceil is the integer part operator.

Figure 1 shows an example of the evaluation process. Through changes in the mask, the generator produces changes in the optimum position, and using different values for $\tau$ y $\rho$, we can control the frequency and severity of the changes, respectively. High values of $\rho$ imply more severe changes, while low values of $\tau$ mean more frequent changes.

As stated before, using this idea of masking, any binary encoded static problem can be converted to its dynamic version. In what follows, the base static problems used here are described.

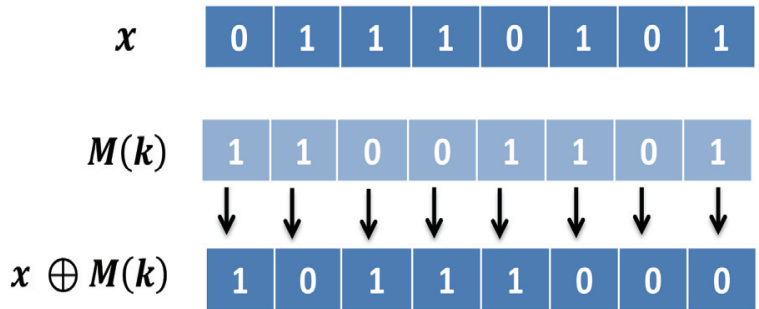

Fig. 1. Given the current mask $M(k)$ and a solution $x$, the objective function is applied to $f(x \oplus M(k))$, where $\oplus$ is the XOR (exclusive OR) operator.

\subsubsection{Knapsack problem}

The knapsack problem is a well-known NP-Hard combinatorial optimization problem [21]. Given a set of $m$ elements the knapsack problem is described as follows:

$$
\begin{aligned}
& \max f(x)=\sum_{i=1}^{m} p_{i} x_{i} \\
& \text { subject to } \sum_{i=1}^{m} w_{i} x_{i} \leqslant C \quad x_{i} \in\{0,1\} i=1 \ldots m
\end{aligned}
$$

where $x=\left(x_{1}, \ldots, x_{m}\right)$ and $x_{i}=1$ if object $i$ is selected or $x_{i}=0$, otherwise. Values $p_{i}$ and $w_{i}$ represent the profit and weight of object $i$, respectively, and $C$ is the capacity of the knapsack. It is believed that knapsack is one of the easiest NP-Hard problems. Several exact algorithms are available and for them, the hardness of random instances, increases with the correlation between weights and profits [34].

Our test instance has $m=100$ objects, and the weights, benefits and capacity are defined as:

$$
\begin{array}{r}
w_{i}=U(1,50) \\
p_{i}=w_{i}+U(1,5) \\
C=0.6 * \sum_{i=1}^{m} w_{i}
\end{array}
$$

where $U(a, b)$ is a function returning a uniformly distributed random value in the $[a, b]$ interval. The definition for $w_{i}$ y $p_{i}$ led to an instance with strong correlation between both values. As stated in [34]:

The strongly correlated instances are hard to solve for two reasons: (a) The instances are illconditioned in the sense that there is a large gap between the continuous and integer solution of the problem; (b) Sorting the items according to decreasing efficiencies correspond to a sorting according to the weights. Thus, for any small interval of the ordered items (i.e. a "core") there is a limited variation in the weights, making it difficult to satisfy the capacity constraint with equality.

Possible unfeasible solutions arising in the search are penalized as in [51]:

$$
f(x)= \begin{cases}\sum_{i=1}^{m} p_{i} x_{i} & \text { if } C^{\prime} \leqslant C \\ 10^{-10} \cdot\left(\left(\sum_{i=1}^{m} w_{i}\right)-C^{\prime}\right) & \text { otherwise }\end{cases}
$$

being $C^{\prime}=\sum_{i=1}^{m} w_{i} x_{i}$ 


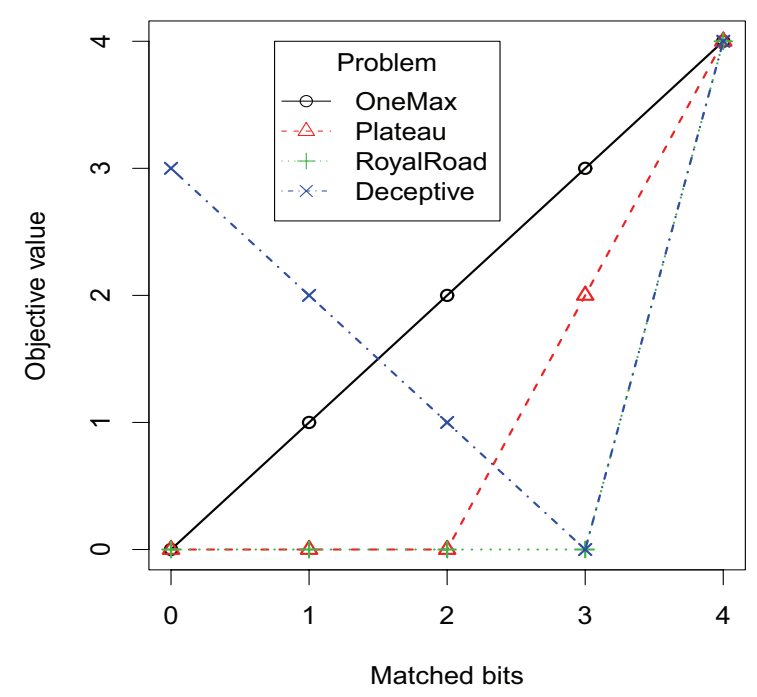

Fig. 2. Fitness contribution of every 4-bits block with respect to the number of correctly matched bits.

\subsubsection{Problems using base binary functions}

We use four additional problems and all of them consist on finding solutions that match all the bits of a target optimal solution. This target solution is initially considered to be the solution where all its bits are set to 1 . To evaluate a solution, we consider blocks of 4 bits where each block contributes a given amount to the final objective value. The contribution of every block of 4 bits depends on the considered functions that are described below:

- OneMax: Each matched bit adds 1 to the fitness.

- Plateau: Three matched bits add 2 to the fitness while four matched bits add 4 and any other amount of bits matched leads to a 0 contribution.

- RoyalRoad: Each perfectly matched block adds 4 to the fitness. Partially matched blocks have fitness 0 .

- Deceptive: Fitness is 4 if all the 4 bits are matched. If not, the fitness for the block is $3 \mathrm{mi}$ nus the number of matched bits.

Figure 2 shows the contribution of every 4-bits block to every function in terms of the number of correctly matched bits.

\subsubsection{Additional information}

The dimension of all the problems was defined as 100 ( 25 blocks of 4-bits for functions described in Section 4.1.2). We considered five different change frequencies $(\tau \in\{1200,3000,6000,9000,12000\}$ fitness function evaluations), and four different severities $(\rho \in\{0.1,0.2,0.5,0.9\})$. The selected $(\tau)$ should be understood as the number of fitness function evaluations allowed between consecutive changes, in other words, it accounts for the number of evaluations that the algorithm has in order to "catch" the optimum until the next movement.

We performed 30 independent runs for every algorithm, problem and combination of $\tau$ and $\rho$, where every run consisted of 100 changes of the fitness function.

\subsection{Reference algorithms}

To better assess the performance of our portfolio, we will perform comparisons against some reference algorithms. In a recent review [7], two algorithms are shown to be competitive in the DOPs considered here: Adaptive Hill Climbing Memetic Algorithm (AHMA) [46] and Self Organized Random Immigrants Genetic Algorithm (SORIGA) [42]. Although there exist more recent methods for the same problems used in this work [13,44], AHMA and SORIGA are still used in comparisons [9, 25, 44], there is a general agreement on their quality, they are easy to understand and more important, their source code is available, thus running them on our benchmarks is much easier. We consider that in this way the comparison is fairer than taking values from published tables.

\subsubsection{Adaptive Hill Climbing Memetic Algorithm (AHMA)}

AHMA, firstly proposed in [46], is essentially a genetic algorithm coupled with a local search that has two neighborhood operators available: Greedy Crossover Hill Climbing (GCHC) and Steepest Mutation Hill Climbing (SMHC). GCHC applies a crossover operator using the elite solution and an individual from the population (chosen by the roulette 
wheel selection). It returns the best child obtained. SMHC operator flips a number of bits in the elite solution (a sort of macro-mutation). The original solution is replaced if the new one is better.

These operators are selected with a probability distribution that is adjusted during the run. After a change, the probability values are kept. AHMA also includes two mechanisms to manage population diversity during the run: Adaptable Dual Mapping (ADM) and Triggered Random Immigrants (TRI).

\subsubsection{SORIGA}

SORIGA (Self Organized Random Immigrants Genetic Algorithm), proposed in [42], is a Genetic Algorithm in which, after the initialization, its population is split into two sub-populations: the main and the secondary one. The best individuals are moved to the main subpopulation whereas the worst individuals are replaced by randomly generated ones ("Random Immigrants") and moved to the secondary subpopulation. Both sub-populations are co-evolved independently until the worst individual considering the two sub-populations belongs to the main one. In this moment, both sub-populations are joined again, evolved for one generation and split in the same way explained before. This process is repeated iteratively until the stopping condition is reached.

\subsubsection{Porfolio Composition}

To implement the algorithms that compose the portfolio we use the Java library BiCIAM [12] which contains standard versions of the most common metaheuristics. For the evaluation of the algorithm portfolio we selected the next methods: BestFirst Hill Climbing, Random Search, Simulated Annealing, Tabu Search, Genetic Algorithm, Evolutionary Strategy and Univariate Marginal Distribution Algorithm. It is important to note that we chose the methods basing just on theirs features (trajectory/population based, search pattern, evolutionary/non-evolutionary method, etc.) and without knowing a priori their performance in the DOPs showed above. We aimed at having a diverse set of algorithm with heterogeneous searching behaviors. Table 3 displays the parameter settings of the methods that integrate the algorithm portfolio. The current version of the Java library BiCIAM, which includes the metaheuristics mentioned above and the implementation of the algorithm portfolio presented in this paper, is available in the next link: http://modo.ugr.es/algorithmportfolio/ index.html

Table 3. Methods that integrate the algorithm portfolio and their parameter settings

\begin{tabular}{|c|c|c|}
\hline Method & Parameter & Setting \\
\hline Hill & hill climbing type & Best-first \\
\hline Climbing & neighborhood operator & one-bit flip mutation \\
\hline Simulated & initial temperature $t_{0}$ & 20 \\
\hline \multirow[t]{5}{*}{ Annealing } & final temperature $t_{n}$ & 0 \\
\hline & number of iterations $\mathrm{T}$ & 50 \\
\hline & $\alpha$ & 0.93 \\
\hline & annealing scheme & $t_{n}=\alpha * t_{0}$ \\
\hline & neighborhood operator & one-bit flip mutation \\
\hline Tabu & tabu list size & 20 \\
\hline \multirow[t]{2}{*}{ Search } & tabu list content & solutions \\
\hline & neighborhood operator & one-bit flip mutation \\
\hline Evolutionary & population size & 50 \\
\hline \multirow[t]{3}{*}{ Strategy } & mutation probability & 0.9 \\
\hline & selection operator & truncation $(20)$ \\
\hline & mutation operator & uniform \\
\hline Genetic & population size & 50 \\
\hline \multirow[t]{5}{*}{ Algorithm } & mutation probability & 0.5 \\
\hline & crossover probability & 0.9 \\
\hline & selection operator & truncation $(20)$ \\
\hline & crossover operator & uniform \\
\hline & mutation operator & uniform \\
\hline Estimation of & population size & 50 \\
\hline Distribution & selection operator & truncation $(20)$ \\
\hline UMDA & probability distribution & UMDA [36] \\
\hline
\end{tabular}

\subsection{Performance measure}

The algorithms will be evaluated using the "offline performance" [4] which is defined as follows:

$$
F_{B G}=\frac{1}{N} \sum_{i=1}^{N}\left(\frac{1}{G} \sum_{e=1}^{G} F_{B_{i e}^{k}}\right)
$$

where $N$ is the number of runs; in the original definition, $G$ stands for the number of generations (which has perfect sense when talking about evolutionary 
algorithms) but here, $G=100 \cdot \tau$ is the number of fitness function evaluations allowed for the portfolio in one run; the value $k=\lceil e / \tau\rceil, k \in\{0 \ldots 100\}$ is the current change period; and $F_{B_{i}}^{k}$ is the fitness of the best solution found in the $k$-th change period of the $i$-th run up to the $e$-th evaluation (or $(e-(k-1) \tau)$-th evaluation of the $k$-th change period).

\subsection{Statistical assessment of results}

Nowadays, it is widely assumed that any comparison among a set of algorithms over a set of problems should be supported by statistical testing. In this article we follow the guidelines proposed in [15] where non-parametric statistical testing is suggested in situations like the one faced in this contribution (several problems, algorithms and configurations).

Firstly, we will apply Friedman's test to check if significant differences exist among a set of algorithms. Besides this, Friedman's average rank allows to sort the algorithms in terms of performance. Secondly, if such significant differences are detected, we use Wilcoxon's test for pairwise comparisons of algorithms, and Holm's and Finner's posthoc tests for one-to-many comparisons between the best algorithm (as indicated by Friedman's rank output) and the rest.

Commercial software SPSS was used for Friedman's and Wilcoxon's tests and KEEL tool [15] for Holm's and Finner's post-hoc tests.

\section{Results}

The computational experiments are oriented to analyze the following questions:

- Does the portfolio obtain better results when using a learning scheme than when not? Which variant of the learning scheme leads to better results? How does the learning scheme influence the selection of the algorithms?

- Does the best portfolio learning variant obtain better results than the individual components when they are run isolated?

- How the performance of the algorithm portfolio is with respect to good reference algorithms (i.e. SORIGA and AHMA)?
Every algorithm in this contribution is analyzed over 5 problems (One-Max, Plateau, Royal-Road, Deceptive and Knapsack), 4 levels of severity $(\rho)$ and 5 different change frequency $(\tau)$, that is, it is tested over a total of 100 problem configurations. For the sake of simplicity and understanding, the offline performance obtained by the different methods, in each problem configuration considered in this experimentation, is not displayed during the analysis of the results. The interested reader can refer to Appendix 1 to check this information.

\subsection{Analysis of the learning scheme}

In this subsection we will analyze the learning schemes presented in Section 3.1 to check if their use makes sense (if they lead to better results than an algorithm portfolio without learning scheme), which of them obtains the best performance and how the learning scheme influences the selection of the algorithms.

\subsubsection{Learning vs No Learning}

The aim of this first analysis is to verify whether or not, the learning schemes proposed lead the portfolio to obtain better results than a strategy without learning (AP-NoLearn), i.e. a portfolio where the constituent methods have the same selection probability along the whole run.

In first place we compare, using Wilcoxon's test at a significance level $\alpha=0.05$, every learning scheme against AP-NoLearn and the results are shown in Table 4. The first column indicates the learning scheme under consideration. The second column "Global" states the result of the comparison over all the problems and configurations. The rest of the columns correspond to the results in every problem. The ' $>$ ' sign states that the considered scheme is statistically better than AP-NoLearn, ' $<$ ' means the opposite, and ' - ' indicates the difference in performance is not significant.

Considering the "Global" column, just half of the learning schemes tested lead to significantly better performance against AP-NoLearn, thus making relevant the need of a careful design of the learning strategy. In other words, a bad learning strategy may lead 
Table 4. Learning vs. no learning. Results of the pairwise comparisons, using the Wilcoxon's nonparametric test at a significance level of $\alpha=0.05$, between the learning schemes proposed and the variant of the algorithm portfolio without learning scheme (AP-NoLearn). The first column indicates the credit assignment scheme under consideration. The second column states the result of the comparison over all the problems and configurations. The rest of the columns correspond to the results in every problem. The ' $>$ ' sign states that the considered scheme is statistically better than AP-NoLearn, ' $<$ ' means the opposite, and ' - ' indicates that the difference in performance is not significant.

\begin{tabular}{c|c|c|c|c|c|c}
\hline Learning scheme & Global & OneMax & Plateau & RoyalRoad & Deceptive & Knapsack \\
\hline RS-AP-RB & $>$ & $>$ & $>$ & $>$ & $>$ & $>$ \\
\hline RS-AP-REB & - & $>$ & $>$ & $>$ & - & - \\
\hline RS-IP-RB & $>$ & $>$ & $>$ & $>$ & - & $>$ \\
\hline RS-IP-REB & $<$ & - & - & $<$ & $<$ & $>$ \\
\hline NRS-AP-RB & $<$ & $<$ & $<$ & - & - & - \\
\hline NRS-AP-REB & $>$ & $>$ & $>$ & $>$ & - & $<$ \\
\hline NRS-IP-RB & $>$ & $>$ & $>$ & $>$ & - & $>$ \\
\hline NRS-IP-REB & $<$ & $<$ & $<$ & $<$ & $<$ & $>$ \\
\hline
\end{tabular}

to worse results than having no learning. It is not clear which component of the learning scheme have a higher impact on the performance, although those schemes using 'RB' (giving reward when the generated solution is strictly better than the reference one) provide better results.

Considering the results disaggregated by problem, the cases for OneMax, Plateau and RoyalRoad are quite similar to those in the Global case. Besides, the signs in the table are almost equal among the three problems, probably meaning that the portfolio behaves similarly on them. Deceptive is perhaps the most complex problem and its intrinsic structure "confuses" the learning scheme. Only RS-AP$\mathrm{RB}$ is able to obtain better results than AP-NoLearn. When considering the Knapsack problem, perhaps the one closer to real life problems, the learning feature starts to be very relevant. Just in one case out of eight (NRS-AP-REB) the use of learning returned worst results than no learning. The other seven cases allowed obtaining better or equal results than AP-NoLearn. It has to be noted that RS-AP$\mathrm{RB}$ is the only variant that consistently outperforms AP-NoLearn over all the cases considered.

\subsubsection{Analysis of learning schemes}

We will compare here the different learning schemes proposed to determine what it is the best one. We have analyzed the best scheme both globally (over all problems) and on each specific problem. Firstly, and using the Friedman test, we compared all learning strategies over all problems and on each problem separately. In all cases the test returned a $p-$ value $=0$, thus indicating that there are significant differences among the portfolios when using different learning schemes.

As we stated before, Friedman's test also outputs a mean ranking for each compared method. These results are shown in Figure 3 where each series represents the mean raking for a specific learning scheme on each of the cases mentioned before (Global, OneMax, Plateau, RoyalRoad, Deceptive and Knapsack). In order to assess whether the best learning strategy on each case has a performance significantly different to the others, we applied the Holm's and Finner's post-hoc tests at a significance level of $\alpha=0.05$. Table 5 shows the results of these tests, where the symbol ' $*$ ' indicates the learning scheme considered as control method (the method with the best mean ranking according to Friedman's test), whereas ' $>$ ' and '-' indicates the existence or not, respectively, of significant differences between the control method and the corresponding learning scheme. In case both tests do not provide the same result (e.g., Holm's test does not rejects the hull hypothesis and Finner's does), the result of the Finner's post-hoc test is displayed within parenthesis. 


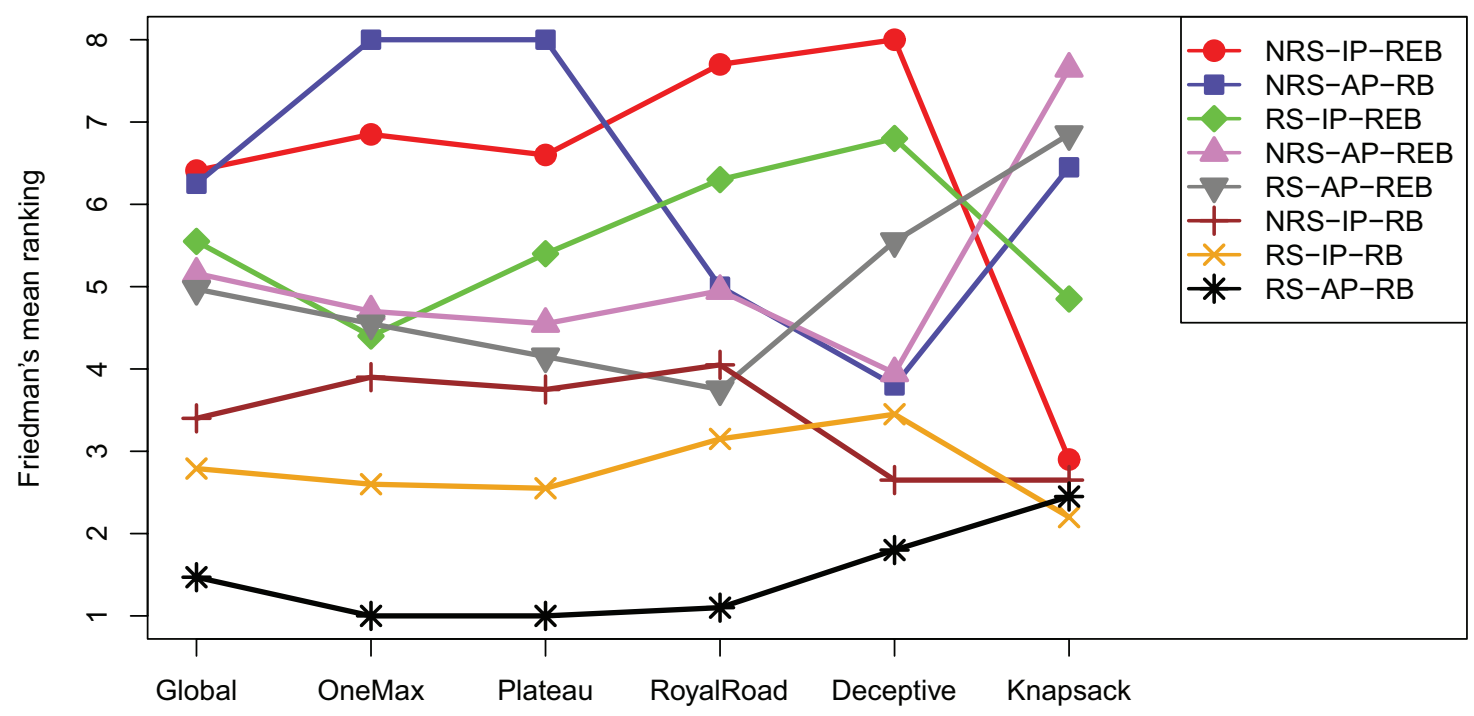

Fig. 3. Mean ranking provided by the Friedman's nonparametric test when all learning schemes are compared. Each series represents the mean raking ( $\mathrm{Y}$ axis) for a specific learning scheme when all problems are considered (Global) and for each problem (OneMax, Plateau, RoyalRoad, Deceptive and Knapsack).

Figure 3 shows that the best ranked scheme globally is RS-AP-RB (credit is restarted after a change, penalization is active and credit is gained if the new solution is strictly better than the best one available). Furthermore, the difference in performance with respect to all the other schemes is significant (Table 5). Therefore, RS-AP-RB is statistically the best learning scheme over all problems.

If we consider each problem separately, RS-AP$\mathrm{RB}$ is the best portfolio variant in four out of the five problems, that is, all but the Knapsack problem. Looking at Table 5, it improves significantly the rest of methods in three of these four problems (OneMax, Plateu and RoyalRoad), whereas in Deceptive the null hypothesis cannot be rejected for two learning schemes, RS-AP-RB and NRS-IP-REB (or only NRS-IP-REB if we consider the Finner's post-hoc test). In the Knapsack problem, despite not being the best learning scheme, RS-AP-RB is not significantly worse than RS-IP-RB, the control method in this case. In short, the performance of RS-AP-RB is better or similar to the other learning schemes, so we can conclude that it is the best portfolio variant.

\subsubsection{Analyzing the influence of the learning scheme in the selection of the algorithms}

So far, we have focused on the performance of the different learning schemes. In this section we aim at studying how the learning scheme influences the behavior of the algorithm portfolio. More specifically, we want to analyze how the selection of the algorithms varies from one learning scheme to another by studying the evolution of the selection probabilities $P_{\text {selec }}^{a_{i}}$ of the algorithms that compose the portfolio: Hill Climbing (HC), Random Search (RndS), Simulated Annealing (SA), Tabu Search (TS), Genetic Algorithm (GA), Evolutionary Strategy (ES) and Estimation of Distribution Algorithm (EDA). For the sake of the simplicity and space, we have limited our analysis to two representative learning schemes and problem configurations. Concretely, we have chosen the best learning scheme, RS-AP$\mathrm{RB}$, and its non-restarting counterpart, NRS-AP$\mathrm{RB}$, in order to have a clear view of the effects of restarting the credit after each change. Regarding the problem configurations, we considered the two 
Table 5. Results returned by the Holm's and Finner's post-hoc tests at a significance level of $\alpha=0.05$ when the best learning scheme globally (over all problems) and on each specific problem is compared against the others. The symbol ' $*$ ' indicates the learning scheme considered as control method (the method with the best mean ranking); ' $>$ ' means that the control method improves significantly the corresponding learning scheme; and '-' indicates no significant differences between the two learning schemes. In case both tests do not provide the same result (e.g., Holm's test does not rejects the hull hypothesis and Finner's does), Finner's post-hoc test result is displayed within parenthesis.

\begin{tabular}{c|c|c|c|c|c|c}
\hline Learning scheme & Global & OneMax & Plateau & RoyalRoad & Deceptive & Knapsack \\
\hline RS-AP-RB & $*$ & $*$ & $*$ & $*$ & $*$ & - \\
\hline RS-AP-REB & $>$ & $>$ & $>$ & $>$ & $>$ & $>$ \\
\hline RS-IP-RB & $>$ & $>$ & $>$ & $>$ & $-(>)$ & $*$ \\
\hline RS-IP-REB & $>$ & $>$ & $>$ & $>$ & $>$ & $>$ \\
\hline NRS-AP-RB & $>$ & $>$ & $>$ & $>$ & $>$ & $>$ \\
\hline NRS-AP-REB & $>$ & $>$ & $>$ & $>$ & $>$ & $>$ \\
\hline NRS-IP-RB & $>$ & $>$ & $>$ & $>$ & - & - \\
\hline NRS-IP-REB & $>$ & $>$ & $>$ & $>$ & $>$ & - \\
\hline
\end{tabular}

most representative problems from a practical point of view, RoyalRoad and Knapsack, with intermediate values for severity and frequency of change, concretely, 0.5 and 6000 , respectively.

Figure 4 displays the evolution of the mean selection probability for each algorithm, measured every 600 evaluations and aggregated over 30 runs, in the first five changes of the objective function. Rows and columns corresponds to learning schemes (RSAP-RB and NRS-AP-RB) and problem configurations (RoyalRoad and Knapsack), respectively. In each plot, the horizontal line marks the probability value for a uniform distribution where all individual algorithms have the same selection probability, whereas the vertical lines show when the changes take place.

First, we focus our analysis on the Royal Road configuration. For a better understanding of the analysis, it is important to highlight that in this problem configuration, RS-AP-RB is significantly better than NRS-AP-RB (Mann-Whitney's U nonparametric test $\alpha<0.05$ ) and the performance of the isolated algorithms in descent order (better $\rightarrow$ worse) according to their offline performance is HC, ES, GA, SA, RS, TS and EDA. Looking at the plot we can clearly observe the differences between restarting or not the credit after changes. In the NRS-AP-RB scheme, the probabilities converge after the first change to a virtually uniform distribution, where all the algorithms have the same chances of being selected. However, in the RS-
AP-RB scheme, the probabilities vary right after each change and converge approximately after 3000 evaluations, that is, at half of the period between changes. Focusing on the individual methods, in the period before the first change, $\mathrm{HC}$ presents the highest probability for both credit assignment schemes. In the next stages, we observe very interesting behaviors in RS-AP-RB. The selection probability for $\mathrm{HC}$ becomes closer to the uniform distribution value but experiences abrupt changes for ES, SA and EDA, with high values at similar moments of the stationary periods of the function. Taking into account that SA and EDA are not among the best performing methods for this problem configuration when run individually, this behavior shows that although a solver may not have a good isolate performance, it can be very useful in some moments of the search when it is combined with other methods. This also explains why "forgetting" the learning gained after each change (by restarting the credit) is beneficial in this case. It allows taking advantage of those algorithms that only have a good performance in specific parts of the search but bad in the others.

The evolution of the selection probabilities for the Knapsack configuration is different but it keeps some similarities. In this case, RS-AP-RB is also significantly better than NRS-AP-RB (MannWhitney's U non-parametric test $\alpha<0.05$ ), and the performance of the algorithms in descent order according to their offline performance is SA, HC, RS, TS, GA, EDA y ES. The first issue to highlight here 

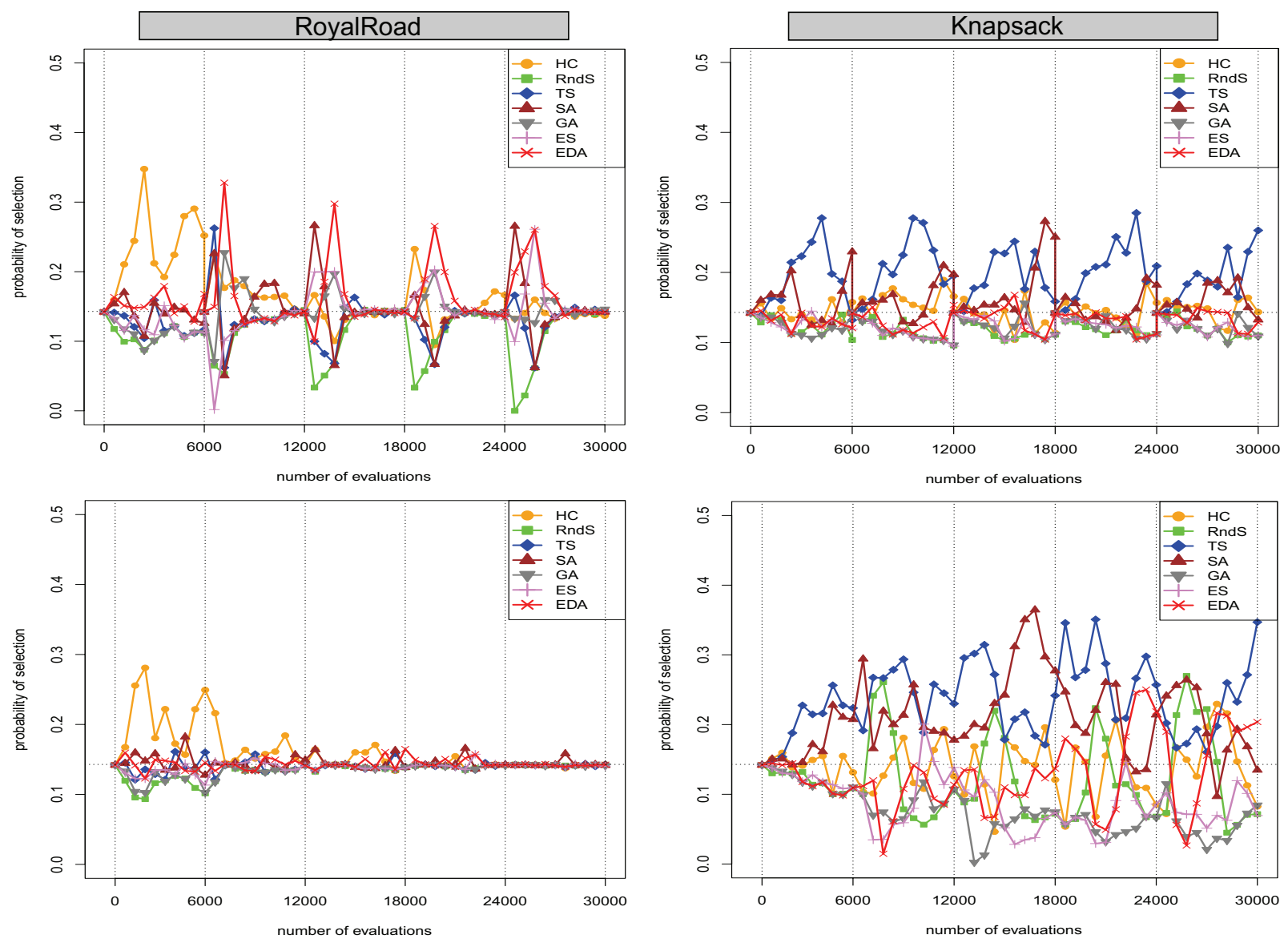

Fig. 4. Evolution of the mean selection probability for each individual algorithm, measured every 600 evaluations and aggregated over 30 runs, in the first five changes of the objective function. Rows and columns corresponds to learning schemes (RS-AP-RB and NRS-AP-RB) and problem configurations ([RoyalRoad (severity 0.5 , frequency of change 6000) and Knapsack (severity 0.5, frequency of change 6000)), respectively. In each plot, the horizontal line marks the probability value for a uniform distribution where all individual algorithms have the same selection probability, whereas the vertical lines show when the changes take place. 


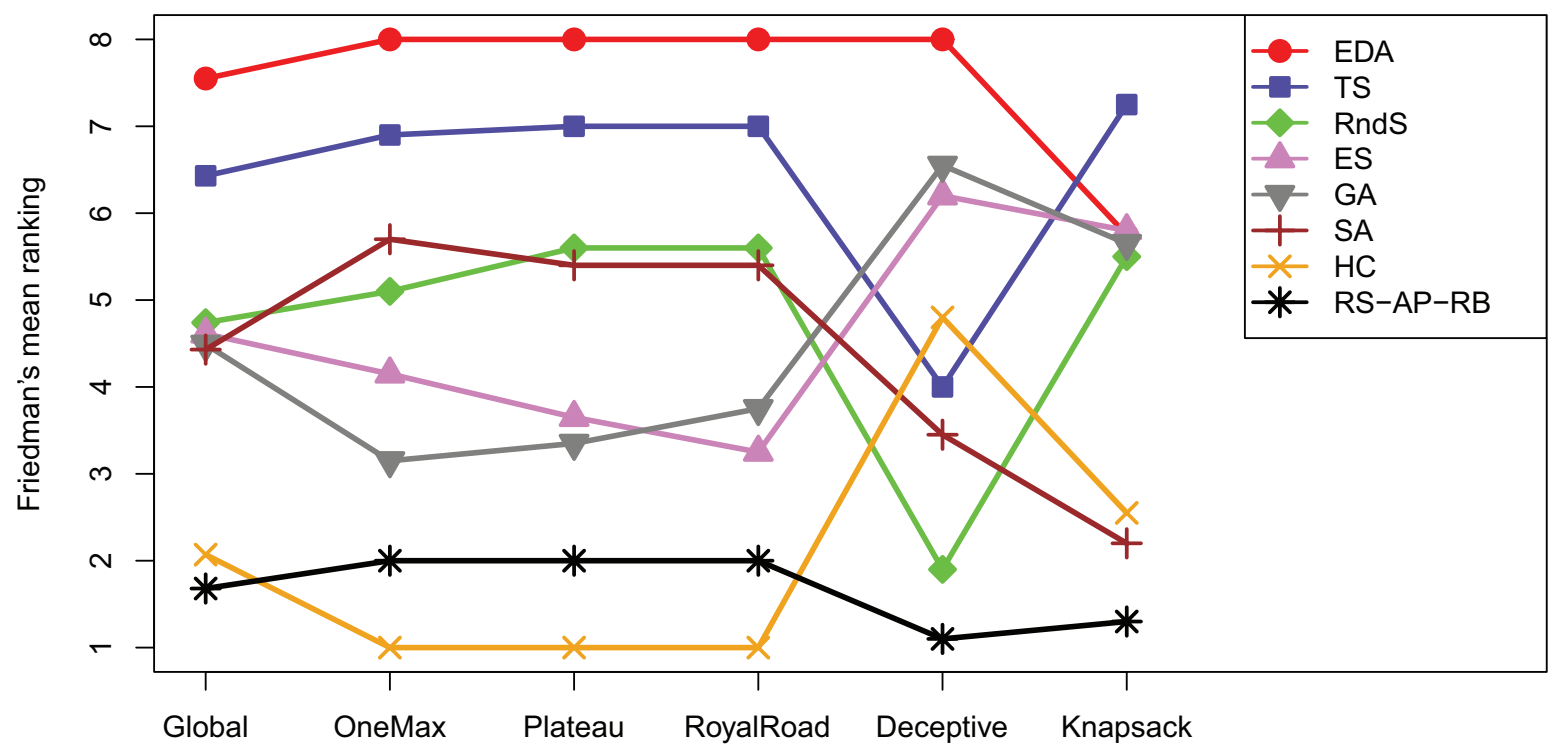

Fig. 5. Mean ranking provided by the Friedman's nonparametric test when the best portfolio variant (RS-AP-RB) is compared against the individual metaheuristics. Each series represent the mean raking (Y axis) for a method when all problems are considered (Global) and for each problem (OneMax, Plateau, RoyalRoad, Deceptive and Knapsack).

is the higher variation of the selection probabilities along the whole stationary periods, unlike in Royal Road where probabilities tended to converged. This is due to the greater difficulty of the Knapsack problem, which slows the convergence of the methods and spread the improvements, and thus the credit rewards, along the whole search process. Analyzing the individual methods, we observe that TS and SA are the algorithms that present a higher selection probability for both learning schemes. Again, it is interesting to see that TS is one of the two algorithms with the highest selection probability despite being the fourth best performing method when it is run individually. This suggests again that bad individual methods could be useful in some part of the search when combined with other algorithms.

\subsection{Comparisons with isolated methods}

In last section we determined the best learning scheme (RS-AP-RB). Here, we will check if the portfolio is able to obtain better results than their in- dividual metaheuristics separately. To this end, we compare RS-AP-RB against the methods that compose the portfolio.

We will follow the same methodology employed in the former section. Figure 5 displays the mean ranking returned by the Friedman's non-parametric test for all methods both globally and on each problem. The $p-$ value obtained in all cases was equal to 0 so we can reject the null hypothesis. We also applied Holm's and Finner's post-hoc tests, at a significance level of $\alpha=0.05$, in order to check if the difference in performance between the best method against the remaining algorithms is significant or not. These results are shown in Table 6 (the notation is the same explained above).

Globally over all problems, RS-AP-RB improves significantly all the isolated methods except $\mathrm{HC}$ (see Figure 5 and Table 6). Analyzing the problems separately, the first issue to highlight is the performance variability of the isolated metaheuristics, especially when the difficulty of the problems increases. Three good examples are $\mathrm{HC}$, RndS and SA, the only 
Table 6. Results returned by the Holm's and Finner's post-hoc tests at a significance level of $\alpha=0.05$ when the best learning scheme (RS-AP-RB) and the individual metaheuristics are compared globally and on each specific problem. The symbol ' $*$ ' indicates the learning scheme considered as control method (the method with the best mean ranking); ' $>$ ' means that the control method improves significantly the corresponding learning scheme; and '-' indicates no significant differences between the two learning schemes. In case both tests do not provide the same result (e.g., Holm's test does not rejects the hull hypothesis and Finner's does), Finner's post-hoc test result is displayed within parenthesis.

\begin{tabular}{c|c|c|c|c|c|c}
\hline Method & Global & OneMax & Plateau & RoyalRoad & Deceptive & Knapsack \\
\hline HC & - & $*$ & $*$ & $*$ & $>$ & - \\
\hline RndS & $>$ & $>$ & $>$ & $>$ & - & $>$ \\
\hline SA & $>$ & $>$ & $>$ & $>$ & $>$ & - \\
\hline TS & $>$ & $>$ & $>$ & $>$ & $>$ & $>$ \\
\hline GA & $>$ & $>$ & $>$ & $>$ & $>$ & $>$ \\
\hline ES & $>$ & $>$ & $>$ & $>$ & $>$ & $>$ \\
\hline EDA & $>$ & $>$ & $>$ & $>$ & $>$ & $>$ \\
\hline RS-AP-RB & $*$ & - & - & - & $*$ & $*$ \\
\hline
\end{tabular}

methods that are not significantly worse than RSAP-RB in all problems. HC and RndS have an opposite performance. While HC offers good results for OneMax, Plateau, RoyalRoad and Knapsack, and poor for Deceptive, RndS performs very well in Deceptive and poorly in the other four problems. As for SA, it shows a high performance for Knapsack and Deceptive but low for OneMax, Plateu and RoyalRoad. On the contrary, the portfolio presents very robust results along all problems. Particularly for the two hardest ones (Knapsack and Deceptive) where it improves all the individual metaheuristics in terms of mean ranking, although the null hypothesis cannot be rejected for RndS in Deceptive, and for HC and SA in Knapsack. In the end, although $\mathrm{HC}$ has a better performance than RS-AP-RB in OneMax, Plateau and RoyalRoad, we can affirm that the best variant of the portfolio obtains similar or significantly better results (in at least one problem) than the isolated versions of the metaheuristics that integrate it.

\subsection{Comparisons against SORIGA and AHMA}

To finish the analysis of the results, we will compare RS-AP-RB against SORIGA and AHMA (both described in Section 4.2), to check whether its results are competitive with high-performance algorithms for these problems. We used the same benchmarks as before and the parameter setting of both methods was done according to the guidelines given in their original works ( [42] and [46], respectively).

We will follow the same comparison scheme used in the two former sections, that is, we will use the mean ranking provided by the Friedman's non-parametric test, which rejects the null hypothesis in all cases $(p-$ value $=0)$, and the Holm's and Finner's post-hoc tests at a confidence level of $\alpha=0.95$ to check the significance of the difference in performance among the three methods. These results are displayed in Figure 6 and Table 7, respectively.

When considering all the problems and configurations (Global), the portfolio coupled with the RSAP-RB learning scheme achieves significantly better performance than AHMA and SORIGA. Separating the results by problem, we observe that RSAP-RB is the best alternative in OneMax, Plateau and RoyalRoad, as shown in Figure 6. Furthermore, the null hypothesis of similar performance can be rejected for both AHMA and SORIGA in the three cases (Table 7). For Deceptive, AHMA is significantly the best method, whereas for Knapsack, AHMA and RS-AP-RB achieved similar performance. Overall, (and setting apart Deceptive, maybe the one most distant to real problems), it becomes clear that RS-AP-RB is better or equal than the reference algorithms considered. 


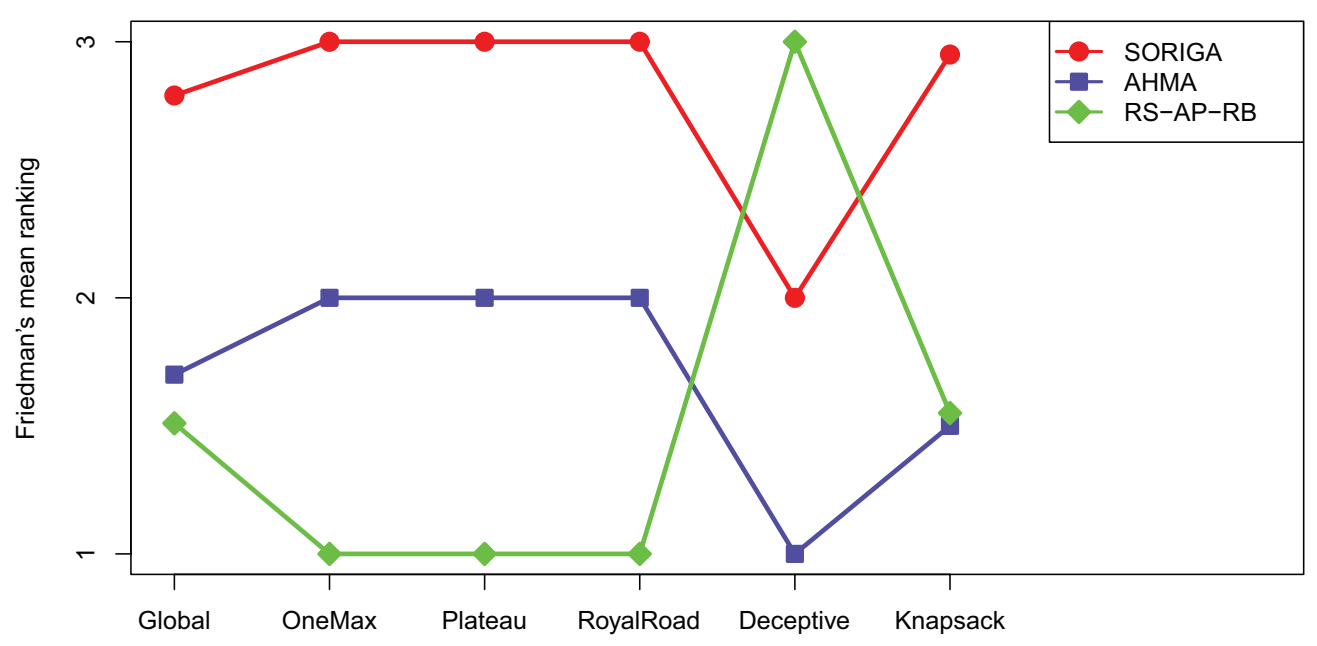

Fig. 6. Mean ranking provided by the Friedman's nonparametric test when the best portfolio variant (RS-AP-RB) is compared against SORIGA and AHMA. Each series represent the mean raking (Y axis) for a specific method when all problems are considered (Global) and for each problem (OneMax, Plateau, RoyalRoad, Deceptive and Knapsack).

Table 7. Results returned by the Holm's and Finner's post-hoc tests at a significance level of $\alpha=0.05$ when the best learning scheme (RS-AP-RB), SORIGA and AHMA are compared globally and on each specific problem. The symbol ' $*$ ' indicates the learning scheme considered as control method (the method with the best mean ranking); ' $>$ ' means that the control method improves significantly the corresponding learning scheme; and '-' indicates no significant differences between the two learning schemes. In case both tests do not provide the same result (e.g., Holm's test does not rejects the hull hypothesis and Finner's does), Finner's post-hoc test result is displayed within parenthesis.

\begin{tabular}{c|c|c|c|c|c|c}
\hline Method & Global & OneMax & Plateau & RoyalRoad & Deceptive & Knapsack \\
\hline RS-AP-RB & $*$ & $*$ & $*$ & $*$ & $>$ & - \\
AHMA & $>$ & $>$ & $>$ & $>$ & $*$ & $*$ \\
SORIGA & $>$ & $>$ & $>$ & $>$ & $>$ & $>$ \\
\hline
\end{tabular}




\section{Conclusions}

In this work we have presented an Algorithm Portfolio to solve combinatorial Dynamic Optimization Problems. This method consists of a set of metaheuristics that are run iteratively. At each stage of the search, the portfolio selects which metaheuristic to apply using a credit based approach that acts as a learning scheme.

The algorithm portfolio was tested over five test problems (OneMax, Plateau, RoyalRoad, Deceptive and Knapsack) to which we induced dynamism by means of XOR-DOP generator. For each problem we considered 4 and 5 different severities and frequencies of change, respectively. To compare the methods, we employed the offline performance, as performance measure, and paired non-parametric tests to check the significance of the differences among algorithms.

The experimentation was oriented to check: whether the algorithm portfolio obtained better results when using a learning scheme than when not; what learning scheme provided better results; how the learning scheme influenced the selection of algorithms; if the portfolio with the best learning scheme improved the performance of the individual metaheuristics that compose it; and how the performance of the portfolio was with respect to two reference methods in the literature, AHMA and SORIGA.

After analyzing the results of the experimentation we can draw the next conclusions:

- The right design of the learning scheme for the algorithm portfolio is a crucial task in DOPs, since only 4 out of 8 learning schemes provided better result than the non-learning version of the algorithm portfolio.

- Different learning schemes lead to different patterns of algorithm selection.

- Algorithms with bad performance when run individually may be useful in specific moments of the search when combined with other methods.

- RS-AP-RB was the only learning scheme that consistently outperformed the non-learning version of the algorithm portfolio in the five problems considered.
- RS-AP-RB was significantly the best credit assignment scheme both globally and in the majority of the problems, except for Knapsack. We understand the lower performance of RS-AP-RB in knapsack problem, as an indication that the structure of the problem may influence the performance of the learning scheme, making some features more appropriate than others (e.g., penalization performs better for OneMax, Plateau, RoyalRoad and Deceptive, while worse for the Knapsack problem).

- When considering the results over all problems, RS-AP-RB improved all the individual metaheuristics that integrate it, with all the difference in performance significant but one, Hill Climbing. Hill Climbing is the best standalone method for OneMax, Plateau and RoyalRoad. As a consequence, RS-AP-RB selects this method with a higher frequency, making it, at the end, to reach a Hill Climbing-like behavior. The situation is different in Deceptive and Knapsack problems, where the differences in performance are lower among the individual methods. Thus RS-AP-RB shows a behavior that can be understood as a "hybrid" among its constituent methods, taking the "best" of each one.

- RS-AP-RB offered very robust results in all problems, in contrast to the performance variability of the individual metaheuristics.

- Over all problems, RS-AP-RB obtained significantly better results than AHMA and SORIGA.

In general terms, the results showed that the old idea of the portfolio of algorithms can also provide good results in DOPs. An important aspect to highlight it is the extreme simplicity of the learning scheme of the portfolio and the metaheuristics that integrate it. We have seen how very general and basic versions of common metaheuristics working together under a simple learning scheme can provide competitive results with respect to ad-hoc highperformance methods for DOPs. In our opinion, these results show that algorithm portfolios could be a good paradigm for designing solvers not only for static problems but also for dynamic ones and therefore, they deserve a greater attention in this field. 


\section{References}

1. P. Angeline. Tracking extrema in dynamic environments. In P. Angeline, R. Reynolds, J. McDonnell, and R. Eberhart, editors, Evolutionary Programming VI, volume 1213 of Lecture Notes in Computer Science, pages 335-345. Springer Berlin Heidelberg, 1997.

2. T. Blackwell and J. Branke. Multiswarms, exclusion, and anti-convergence in dynamic environments. IEEE Transactions on Evolutionary Computation, 10(4):459-472, 2006.

3. J. Branke, M. Orbayi, and S. Uyar. The role of representations in dynamic knapsack problems. In F. Rothlauf, J. Branke, S. Cagnoni, E. Costa, C. Cotta, R. Drechsler, E. Lutton, P. Machado, J. Moore, J. Romero, G. Smith, G. Squillero, and H. Takagi, editors, Applications of Evolutionary Computing, volume 3907 of Lecture Notes in Computer Science, pages 764-775. Springer Berlin Heidelberg, 2006.

4. J. Branke and H. Schmeck. Designing evolutionary algorithms for dynamic optimization problems. In A. Ghosh and S. Tsutsui, editors, Advances in Evolutionary Computing, Natural Computing Series, pages 239-262. Springer Berlin Heidelberg, 2003.

5. E. K. Burke, M. Gendreau, M. Hyde, G. Kendall, G. Ochoa, E. Özcan, and R. Qu. Hyper-heuristics: A survey of the state of the art. Journal of the Operational Research Society, 64(12):1695-1724, 2013.

6. P. Cowling, G. Kendall, and E. Soubeiga. A hyperheuristic approach to scheduling a sales summit. In E. Burke and W. Erben, editors, Practice and Theory of Automated Timetabling III, volume 2079 of Lecture Notes in Computer Science, pages 176-190. Springer Berlin Heidelberg, 2001.

7. C. Cruz, J. R. González, and D. A. Pelta. Optimization in dynamic environments: a survey on problems, methods and measures. Soft Computing, 15(7):14271448, 2011.

8. F. O. de França, F. J. Von Zuben, and L. N. de Castro. An artificial immune network for multimodal function optimization on dynamic environments. In Proceedings of the 2005 Conference on Genetic and Evolutionary Computation, GECCO '05, pages 289-296, New York, NY, USA, 2005. ACM.

9. I. G. del Amo, D. A. Pelta, J. R. González, and A. D. Masegosa. An algorithm comparison for dynamic optimization problems. Applied Soft Computing, 12(10):3176 - 3192, 2012.

10. S. Droste. Analysis of the (1+1) EA for a dynamically bitwise changing OneMax. In E. Cantú-Paz, J. Foster, K. Deb, L. Davis, R. Roy, U.-M. O’Reilly, H.-G. Beyer, R. Standish, G. Kendall, S. Wilson, M. Harman, J. Wegener, D. Dasgupta, M. Potter, A. Schultz, K. Dowsland, N. Jonoska, and J. Miller, editors, Genetic and Evolutionary Computation (GECCO 2003), volume 2723 of Lecture Notes in Computer Science, pages 909-921. Springer Berlin Heidelberg, 2003.

11. W. Du and B. Li. Multi-strategy ensemble particle swarm optimization for dynamic optimization. Information Sciences, 178(15):3096 - 3109, 2008.

12. J. Fajardo and A. Rosete. Algoritmo multigenerador de soluciones, para la competencia y colaboración de generadores metaheurísticos. Revista Internacional de Investigación de Operaciones, 1:57-63, 2011.

13. C. Fernandes, J. Laredo, A. Rosa, and J. Merelo. The sandpile mutation genetic algorithm: an investigation on the working mechanisms of a diversityoriented and self-organized mutation operator for nonstationary functions. Applied Intelligence, 39(2):279306, 2013.

14. M. Gagliolo and J. Schmidhuber. Learning dynamic algorithm portfolios. Annals of Mathematics and Artificial Intelligence, 47(3-4):295-328, 2006.

15. S. García, D. Molina, M. Lozano, and F. Herrera. A study on the use of non-parametric tests for analyzing the evolutionary algorithms' behaviour: a case study on the CEC'2005 special session on real parameter optimization. Journal of Heuristics, 15(6):617-644, 2009.

16. C. P. Gomes and B. Selman. Algorithm portfolios. Artificial Intelligence, 126:43-62, 2001.

17. J. González, C. Cruz, I. Amo, and D. Pelta. An adaptive multiagent strategy for solving combinatorial dynamic optimization problems. In D. Pelta, N. Krasnogor, D. Dumitrescu, C. Chira, and R. Lung, editors, Nature Inspired Cooperative Strategies for Optimization (NICSO 2011), volume 387 of Studies in Computational Intelligence, pages 41-55. Springer Berlin Heidelberg, 2011.

18. J. R. González, A. D. Masegosa, and I. G. del Amo. A cooperative strategy for solving dynamic optimization problems. Memetic Computing, 3:3-14, 2011.

19. M. Guntsch, M. Middendorf, and H. Schmeck. An ant colony optimization approach to dynamic TSP. In Proceedings of the 2001 Genetic and Evolutionary Computation Conference, GECCO'01, pages 860867, San Francisco, CA, USA, 2001. Morgan Kaufmann.

20. B. A. Huberman, R. M. Lukose, and T. Hogg. An economics approach to hard computational problems. Science, 275:51-54, 1997.

21. H. Kellerer, U. Pferschy, and D. Pisinger. Knapsack Problems. Springer, 2004.

22. B. Kiraz, A. S. Uyar, and E. Özcan. Selection hyper-heuristics in dynamic environments. Journal of the Operational Research Society, 64(12):1753-1769, 2013.

23. C. Li, S. Yang, T. Nguyen, E. Yu, X. Yao, Y. Jin, H. Beyer, and P. Suganthan. Benchmark generator for CEC 2009 competition on dynamic optimization. 
Technical report, 2008.

24. L. Liu, D. Wang, and W. Ip. A permutation-based dual genetic algorithm for dynamic optimization problems. Soft Computing, 13(7):725-738, 2009.

25. A. D. Masegosa, D. Pelta, and I. G. del Amo. The role of cardinality and neighborhood sampling strategy in agent-based cooperative strategies for dynamic optimization problems. Applied Soft Computing, 14, Part C(0):577 - 593, 2014.

26. D. C. Mattfeld and C. Bierwirth. An efficient genetic algorithm for job shop scheduling with tardiness objectives. European Journal of Operational Research, 155(3):616 - 630, 2004.

27. M. Mavrovouniotis and S. Yang. A memetic ant colony optimization algorithm for the dynamic travelling salesman problem. Soft Computing, 15(7):14051425, 2011.

28. R. Morrison. Designing Evolutionary Algorithms for Dynamic Environments. Natural Computing Series. Springer Berlin Heidelberg, 2004.

29. R. Morrison and K. De Jong. A test problem generator for non-stationary environments. In Proceedings of the 1999 Congress on Evolutionary Computation, CEC 1999, volume 3, pages 2047-2053, Washington D.C., USA, 1999. IEEE Press.

30. T. T. Nguyen, S. Yang, and J. Branke. Evolutionary dynamic optimization: A survey of the state of the art. Swarm and Evolutionary Computation, 6:1 - 24, 2012.

31. M. Nikolić, F. Marić, and P. Janičić. Simple algorithm portfolio for SAT. Artificial Intelligence Review, 40(4):457-465, 2013.

32. F. Peng, K. Tang, G. Chen, and X. Yao. Populationbased algorithm portfolios for numerical optimization. IEEE Transactions on Evolutionary Computation, 14(5):782-800, 2010.

33. M. Petrik and S. Zilberstein. Learning parallel portfolios of algorithms. Annals of Mathematics and Artificial Intelligence, 48(1-2):85-106, 2006.

34. D. Pisinger. Where are the hard knapsack problems? Computers and Operations Research, 32(9):22712284, 2005.

35. P. Rohlfshagen and X. Yao. The dynamic knapsack problem revisited: A new benchmark problem for dynamic combinatorial optimisation. In M. Giacobini, A. Brabazon, S. Cagnoni, G. Caro, A. Ekárt, A. Esparcia-Alcázar, M. Farooq, A. Fink, and P. Machado, editors, Applications of Evolutionary Computing, volume 5484 of Lecture Notes in Computer Science, pages 745-754. Springer Berlin Heidelberg, 2009.

36. R. Santana, P. Larrañaga, and J. A. Lozano. Adaptive estimation of distribution algorithms. In C. Cotta, M. Sevaux, and K. Sörensen, editors, Adaptive and Multilevel Metaheuristics, volume 136 of Studies in
Computational Intelligence, pages 177-197. Springer Berlin Heidelberg, 2008.

37. L. Schönemann. The impact of population sizes and diversity on the adaptability of evolution strategies in dynamic environments. In Proceedings of the 2004 Congress on Evolutionary Computation, CEC2004, volume 2, pages 1270-1277, Portland, OR, US, 2004. IEEE Press.

38. L. Schönemann. Evolution strategies in dynamic environments. In S. Yang, Y.-S. Ong, and Y. Jin, editors, Evolutionary Computation in Dynamic and Uncertain Environments, volume 51 of Studies in Computational Intelligence, pages 51-77. Springer Berlin Heidelberg, 2007.

39. N. Shukla, A. Choudhary, P. Prakash, K. Fernandes, and M. Tiwari. Algorithm portfolios for logistics optimization considering stochastic demands and mobility allowance. International Journal of Production Economics, 141(1):146 - 166, 2013.

40. N. Shukla, M. K. Tiwari, and D. Ceglarek. Genetic algorithms based algorithm portfolio for inventory routing problem with stochastic demand. International Journal of Production Research, 51(1):118137, 2013.

41. S. A. Stanhope and J. M. Daida. (1+1) genetic algorithm fitness dynamics in a changing environment. In Proceedings of the 1999 Congress of Evolutionary Computation, CEC 2009, volume 3, pages 18511858, Washington D.C., USA, 1999.

42. R. Tinós and S. Yang. A self-organizing random immigrants genetic algorithm for dynamic optimization problems. Genetic Programming and Evolvable Machines, 8(3):255-286, 2007.

43. H. R. Topcuoglu, A. Ucar, and L. Altin. A hyperheuristic based framework for dynamic optimization problems. Applied Soft Computing, 19(0):236 - 251, 2014.

44. A. M. Turky and S. Abdullah. A multi-population harmony search algorithm with external archive for dynamic optimization problems. Information Sciences, 272(0):84 - 95, 2014.

45. G. Uludağ, B. Kiraz, A. Etaner-Uyar, and E. Özcan. A framework to hybridize PBIL and a hyper-heuristic for dynamic environments. In C. A. C. Coello, V. Cutello, K. Deb, S. Forrest, G. Nicosia, and M. Pavone, editors, Parallel Problem Solving from Nature, PPSN XII, volume 7492 of Lecture Notes in Computer Science, pages 358-367. Springer Berlin Heidelberg, 2012.

46. H. Wang, D. Wang, and S. Yang. A memetic algorithm with adaptive hill climbing strategy for dynamic optimization problems. Soft Computing, 13(8-9):763780, 2009.

47. L. Xu, F. Hutter, H. H. Hoos, and K. LeytonBrown. SATzilla: Portfolio-based algorithm selection for SAT. Journal of Artificial Intelligence Research, 
32:565-606, 2008.

48. S. R. Yadav, R. R. M. Muddada, M. Tiwari, and R. Shankar. An algorithm portfolio based solution methodology to solve a supply chain optimization problem. Expert Systems with Applications, 36(4):8407-8420, 2009.

49. S. Yang, Y. Jiang, and T. T. Nguyen. Metaheuristics for dynamic combinatorial optimization problems. IMA Journal of Management Mathematics, 24(4):451-480, 2013.

50. S. Yang, Y. Ong, and Y. Jin. Evolutionary Com- putation in Dynamic and Uncertain Environments, volume 51 of Studies in Computational Intelligence. Springer, 2007.

51. S. Yang and X. Yao. Experimental study on population-based incremental learning algorithms for dynamic optimization problems. Soft Computing, 9(11):815-834, 2005.

\section{Appendix A}


J. Fajardo, A.D. Masegosa, D. Pelta

Table A.1. Offline performance obtained in 30 runs by each learning schemes considered in the experimentation for each problem, severity and frequency of change.

\begin{tabular}{|c|c|c|c|c|c|c|c|c|c|c|c|c|c|c|c|c|c|c|c|c|c|}
\hline & & \multirow{2}{*}{\multicolumn{4}{|c|}{$\begin{array}{l}\text { OneMax } \\
\text { severity }\end{array}$}} & \multirow{2}{*}{\multicolumn{4}{|c|}{ Plateau }} & \multirow{2}{*}{\multicolumn{4}{|c|}{$\begin{array}{l}\text { RoyalRoad } \\
\end{array}$}} & \multirow{2}{*}{\multicolumn{4}{|c|}{$\begin{array}{l}\text { Deceptive } \\
\text { severity }\end{array}$}} & \multicolumn{4}{|c|}{ Knapsack } \\
\hline & & & & & & & & & & & & & & & & & & & $\mathrm{sev}$ & & \\
\hline & change & 0.1 & 0.2 & 0.5 & 0.9 & 0.1 & 0.2 & 0.5 & 0.9 & 0.1 & 0.2 & 0.5 & 0.9 & 0.1 & 0.2 & 0.5 & 0.9 & 0.1 & 0.2 & 0.5 & 0.9 \\
\hline \multirow[t]{5}{*}{ RS-IP-REB } & 1200 & 96.697 & 93.627 & 88.183 & 88.009 & 92.951 & 85.863 & \begin{tabular}{|l|l|}
73.776 \\
\end{tabular} & \begin{tabular}{|l|l|}
73.967 \\
\end{tabular} & \begin{tabular}{|l|l|}
75.169 \\
\end{tabular} & 59.801 & \begin{tabular}{|l|l|}
44.084 \\
\end{tabular} & \begin{tabular}{|l|l|}
40.929 \\
\end{tabular} & \begin{tabular}{|l|l|}
18.201 \\
\end{tabular} & \begin{tabular}{|l}
17.282 \\
\end{tabular} & \begin{tabular}{|l|l|}
16.710 \\
\end{tabular} & 16.893 & \begin{tabular}{|l|l|}
1674.750 \\
\end{tabular} & \begin{tabular}{|l|l|}
1668.979 \\
\end{tabular} & 1665.183 & 1674.889 \\
\hline & 3000 & 98.650 & 97.330 & 94.934 & 94.932 & 5.861 & 93.046 & 86.141 & 86.149 & \begin{tabular}{|l|l|}
83.193 \\
\end{tabular} & 71.834 & 56.033 & 51.294 & \begin{tabular}{|l|} 
\\
20.123
\end{tabular} & 19.249 & 18.599 & 18.599 & 1679.836 & 1674.244 & 1670.331 & 1679.611 \\
\hline & 6000 & 326 & 8.674 & 97.472 & 7.461 & 3.274 & 95.740 & 91.089 & 1.197 & \begin{tabular}{|l|l|}
87.647 \\
\end{tabular} & 77.650 & \begin{tabular}{|l|l|} 
& 63.426 \\
\end{tabular} & 8.637 & 21.726 & 20.413 & 19.931 & $\mid \begin{array}{l}19.707 \\
\end{array}$ & 1684.083 & 1677.994 & 1674.314 & 1683.923 \\
\hline & 9000 & 99.540 & 99.117 & 98.311 & 98.300 & .789 & 96.696 & 92.894 & 92.975 & 89.458 & 80.805 & 67.657 & 63.931 & 22.679 & 21.388 & 20.829 & 20.702 & 1687.852 & 1680.461 & 1676.542 & 1687.469 \\
\hline & 12000 & 99.662 & 99.339 & 98.731 & 8.724 & .023 & 97.227 & 93.892 & 4.069 & \begin{tabular}{|l}
90.199 \\
\end{tabular} & 81.972 & 70.500 & 7.099 & 23.729 & 22.160 & 21.690 & 21.353 & 1689.696 & 1682.967 & 1678.778 & 1689.932 \\
\hline \multirow[t]{4}{*}{ RS-IP-RB } & 1200 & 97.363 & 94.564 & 87.864 & 87.637 & 94.402 & \begin{tabular}{|l|l|}
87.784 \\
\end{tabular} & 71.548 & \begin{tabular}{|l|l|}
71.467 \\
\end{tabular} & $\begin{array}{l}77.185 \\
\end{array}$ & 60.925 & 43.184 & 40.244 & \begin{tabular}{|l|l|}
19.714 \\
\end{tabular} & \begin{tabular}{|l|l|}
18.985 \\
\end{tabular} & 18.339 & \begin{tabular}{|l|l|}
18.346 \\
\end{tabular} & \begin{tabular}{|l|l|}
1675.406 \\
\end{tabular} & \begin{tabular}{|l|l|}
1669.828 \\
\end{tabular} & 1664.828 & 1675.449 \\
\hline & & 98.942 & 97.811 & 95.086 & 95.024 & 7.736 & 94.774 & 86.920 & 86.983 & 87.960 & 75.308 & 56.465 & 52.667 & \begin{tabular}{|l|}
22.491 \\
\end{tabular} & 21.438 & 20.995 & 20.737 & 1681.025 & 675.253 & 1671.222 & 1681.000 \\
\hline & 6000 & 473 & 98.907 & 97.564 & 7.510 & 3.841 & 97.207 & 92.955 & 93.053 & 92.340 & 82.820 & 66.147 & 63.261 & 24.206 & 23.468 & 22.815 & 22.562 & 1686.174 & 1680.274 & 1676.124 & 1687.073 \\
\hline & 9000 & .649 & 99.271 & 98.370 & 3.328 & 203 & 98.074 & 5.131 & 5.151 & 94.125 & 86.425 & 71.761 & & 25.510 & & 23.928 & 23.675 & 169 & & 50 & 1689.953 \\
\hline \multirow[t]{5}{*}{ RS-AP-REB } & 1200 & 97.321 & 94.353 & \begin{tabular}{|l|l|}
87.360 \\
\end{tabular} & 87.149 & 1.250 & \begin{tabular}{|l|l|}
87.546 \\
\end{tabular} & 71.518 & 71.416 & \begin{tabular}{|l|l|}
77.925 \\
\end{tabular} & 61.276 & \begin{tabular}{|l|l|}
43.467 \\
\end{tabular} & 40.495 & \begin{tabular}{|l|l|}
19.762 \\
\end{tabular} & 18,644 & \begin{tabular}{|l|l|}
17.992 \\
\end{tabular} & \begin{tabular}{|l|l|}
17.596 \\
\end{tabular} & & & & 1672.086 \\
\hline & 3000 & 924 & 97.731 & 94.904 & 4.810 & .624 & 94.602 & 86.541 & 73 & 7.756 & & & & 2.002 & & 20.400 & 20.214 & 764 & & & 1675.079 \\
\hline & 6000 & 462 & 98.865 & 97.441 & .404 & 792 & 97.109 & 2.653 & & 92.218 & & & & 953 & & 22.351 & & & & & \\
\hline & 9000 & 640 & 99.238 & 98.296 & 3.273 & .177 & 97.968 & 4.724 & 65 & 94.030 & 86 & & & 5.454 & & 23.503 & 23.210 & & & & 354 \\
\hline & 12000 & 99.728 & 99.429 & 98.721 & 3.706 & 0.353 & 98.417 & 95.928 & 5.922 & 94.946 & 88.001 & 74.934 & 2.949 & 26.417 & 2 & 24.434 & 24.067 & 73 & 504 & 943 & 1676.417 \\
\hline \multirow[t]{5}{*}{ RS-AP-RB } & 1200 & 532 & 9.266 & \begin{tabular}{|l|l|}
99.617 \\
\end{tabular} & 9.544 & 8.739 & \begin{tabular}{|l|l|}
95.148 \\
\end{tabular} & 86.702 & 23 & \begin{tabular}{|l|l|}
92.048 \\
\end{tabular} & 92.452 & 84 & 448 & 0.195 & & \begin{tabular}{|l|l|}
18.431 \\
\end{tabular} & 18.367 & 46 & 66 & 36 & 534 \\
\hline & & 007 & .096 & 96.215 & 9 & 39 & 95.425 & 年 & 25 & 58 & 77.949 & 39 & 13 & 407 & & 21.060 & 20. & & & & 525 \\
\hline & 6000 & .507 & .047 & 98.105 & 91 & 78 & 97 & 94.667 & 70 & 14 & 40 & 82 & 29 & 4.589 & & 23.006 & 22.973 & 11 & & & 967 \\
\hline & 9000 & 99.670 & 99.363 & 98.740 & 725 & .228 & 98.314 & 96.345 & 6.384 & 55 & 580 & 79.680 & 97 & 17 & 24 & 24.265 & 24.001 & 172 & & & 532 \\
\hline & 12000 & 99.750 & 99.523 & 99.056 & .042 & .429 & 98.720 & 97.231 & 7.194 & .329 & 90.588 & 93.004 & 13 & 7.272 & 25.686 & 25.304 & 24.927 & 908 & 759 & & 1691.020 \\
\hline \multirow{3}{*}{ NRS-IP-REB } & 1200 & & & & & & & & & & & & & & & 86 & 14. & & & & 560 \\
\hline & 9000 & 99.337 & 8.576 & 96.656 & . & 128 & 94 & 9.811 & 91.8 & 7860 & 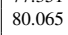 & 5.131 & 75 & 1.020 & 18. & 17.636 & 18. & 68 & & & 168 \\
\hline & 12000 & 99.511 & 98.932 & 97.172 & 97.301 & 3.518 & 95.368 & 90.601 & 92.256 & 88.372 & 81.515 & 68.120 & 51.566 & 22.070 & 19.5 & 17.852 & 18.747 & 1692 & 684.379 & 28 & 1692.290 \\
\hline \multirow[t]{5}{*}{ NRS-IP-RB } & 1200 & Don & 04488 & 86.434 & 86 & (1) & \begin{tabular}{|l|}
87671 \\
\end{tabular} & \begin{tabular}{|l|l|}
69.833 \\
\end{tabular} & & 78607 & + & 43116 & & 20.012 & 191 & \begin{tabular}{|l|l|}
18.463 \\
\end{tabular} & \begin{tabular}{|l|}
18.4 \\
\end{tabular} & 678 & & & 1675.860 \\
\hline & & & 97.791 & 94.466 & 9 & .10 & 94.080 & 306 & & 1 & 75.075 & & & 2.428 & & 21.073 & 20. & & & & \\
\hline & 6000 & 486 & 98.891 & 97.210 & 9 & 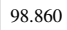 & 97179 & 92.118 & 2.074 & 12 & 83.072 & 65364 & & 4.632 & 33 & 22801 & 22. & & & & 168 \\
\hline & & 559 & 99.259 & 98.147 & & & & 94.44 & 4.4 & 94.110 & & & & 2 & & 33 & & & & & 53 \\
\hline & 12000 & 99.745 & 99.449 & 98.607 & 9 & 9 & 98.481 & 0560 & $\cdots$ & 9 & 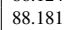 & 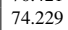 & & 4 & 2 & 4.723 & 24.686 & 69 & 05 & & 1692.137 \\
\hline \multirow{5}{*}{ NRS-AP-REB } & 1200 & 97.441 & 4.457 & 86.2. & 8 & .616 & \begin{tabular}{|l|l|}
87.4 \\
\end{tabular} & 9.53 & 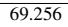 & 8.241 & 6 & 42.6 & 39. & 9.879 & 9.1 & \begin{tabular}{|l|l|}
18.190 \\
\end{tabular} & 18.326 & & & & 1660.947 \\
\hline & 2000 & & (7) & & & & & & & & & & & 2.233 & & & & & & & 1673.837 \\
\hline & 60 & 99.486 & 98.879 & 97.211 & & 9 & 22 & 92.024 & & 2.415 & .778 & 6. & & 4.344 & & 2.818 & 22.360 & 58 & & & 167 \\
\hline & 9000 & 99.657 & 99.257 & & & & & & & & & & & & & & 23814 & & & & 167964 \\
\hline & 12000 & 99.743 & 99.446 & 98.603 & 98.584 & 99.408 & 98.479 & 95.608 & 95.591 & 94.793 & 88.177 & 74.102 & 72.589 & 26.556 & 25.123 & 24.606 & 24.433 & 1678.505 & 669.627 & 637.374 & 1678.866 \\
\hline \multirow[t]{5}{*}{ NRS-AP-RB } & 1200 & & & 73.9 & & & 73.1 & 51.658 & & & & 54.911 & & & & 年 & & & & & 1661 \\
\hline & 3000 & & 81.313 & 81.423 & 79.265 & 75.669 & 64.629 & 62.475 & 61.077 & 76.467 & 109 & & & 21.173 & & & & & & & \\
\hline & 6000 & & & 89 & & & & 80.108 & & & & & & a & 232 & 22.556 & 22.506 & 1677 & & & 1679.441 \\
\hline & 9000 & & 96.288 & 93.037 & 9 & .596 & 92.771 & 86.053 & 86.608 & 91.951 & 83.735 & 71.93 & 72.178 & 26.382 & 25.237 & 24.296 & .495 & & & & 168 \\
\hline & 12000 & 98.700 & 97.442 & 95.130 & 95.303 & 97.428 & 94.571 & 88.966 & 90.208 & 93423 & 87.068 & 77.265 & 77.509 & 27652 & 26108 & 25.800 & 25.670 & 1686.898 & 1682.265 & 1679.556 & 1686.938 \\
\hline
\end{tabular}


Table A.2. Offline performance obtained in 30 runs by the isolated versions of algorithms that compose the portfolio for each problem, severity and frequency of change.

\begin{tabular}{|c|c|c|c|c|c|c|c|c|c|c|c|c|c|c|c|c|c|c|c|c|c|}
\hline & & \multirow{2}{*}{\multicolumn{4}{|c|}{$\begin{array}{l}\text { OneMax } \\
\text { severity }\end{array}$}} & \multirow{2}{*}{\multicolumn{4}{|c|}{$\begin{array}{l}\text { Plateau } \\
\text { severity }\end{array}$}} & \multirow{2}{*}{\multicolumn{4}{|c|}{$\begin{array}{c}\text { RoyalRoad } \\
\text { severity }\end{array}$}} & \multirow{2}{*}{\multicolumn{4}{|c|}{$\begin{array}{l}\text { Deceptive } \\
\text { severity }\end{array}$}} & \multicolumn{4}{|c|}{ Knapsack } \\
\hline & & & & & & & & & & & & & & & & & & & seve & & \\
\hline & change & 0.1 & 0.2 & 0.5 & 0.9 & 0.1 & 0.2 & 0.5 & 0.9 & 0.1 & 0.2 & 0.5 & 0.9 & 0.1 & 0.2 & 0.5 & 0.9 & 0.1 & 0.2 & 0.5 & 0.9 \\
\hline \multirow[t]{5}{*}{$\mathrm{HC}$} & 1200 & \begin{tabular}{|l|}
99.197 \\
\end{tabular} & 98.384 & 95.920 & \begin{tabular}{|l|}
92.639 \\
\end{tabular} & 98.258 & 96.182 & 89.746 & 83.748 & 89.268 & 80.940 & 66.838 & 60.687 & 11.972 & 11.915 & 11.980 & 13.516 & 1676.305 & \begin{tabular}{|l|}
1671.407 \\
\end{tabular} & 1665.839 & 1675.655 \\
\hline & 3000 & 99.682 & \begin{tabular}{|l|l|}
99.348 \\
\end{tabular} & 98.369 & 97.043 & 99.298 & 98.459 & 95.909 & 93.519 & 5.719 & 92.163 & 85.759 & 82.872 & 11.988 & 11.972 & 16.766 & 17.594 & 1677.620 & 1672.574 & 1668.479 & 1677.862 \\
\hline & 6000 & 99.840 & 99.673 & 99.183 & 98.523 & 99.656 & 99.234 & 97.953 & 96.769 & 7.838 & 96.089 & 92.901 & 91.411 & 11.996 & 11.990 & 18.077 & 18.922 & 1678.150 & 1673.188 & 1668.759 & 1679.739 \\
\hline & 9000 & 99.893 & 99.783 & 99.453 & 99.015 & 99.768 & 99.491 & 98.636 & .844 & 566 & 7.411 & 95.307 & 94.281 & 11.997 & 11.992 & 18.665 & 19.271 & 1678.829 & 1673.836 & 1669.775 & 1679.033 \\
\hline & 12000 & 99.920 & 99.837 & 99.592 & 99.259 & 99.829 & 99.619 & 98.981 & 98.372 & 98.926 & 98.041 & 96.461 & 95.708 & 11.999 & 11.995 & 19.137 & 19.456 & 1679.537 & 1673.282 & 1669.751 & 1679.821 \\
\hline \multirow[t]{5}{*}{ RS } & 1200 & \begin{tabular}{|l|}
65.550 \\
\end{tabular} & 64.894 & 64.653 & 64.644 & 39.346 & 38.548 & 38.245 & 38.138 & 24.437 & 24.000 & 23.727 & 23.767 & 18.818 & 18.652 & 18.622 & 18.610 & 1657.082 & \begin{tabular}{|l|}
1656.955 \\
\end{tabular} & 1656.826 & 1656.963 \\
\hline & 3000 & 66.721 & 66.219 & 65.969 & 65.915 & 41.268 & 40.469 & 40.118 & 40.183 & 26.914 & 26.475 & 26.415 & 26.413 & 19.598 & 19.559 & 19.519 & 19.497 & 1663.341 & 1663.146 & 1663.080 & 1663.413 \\
\hline & 6000 & 67.521 & 67.052 & 66.919 & 66.874 & 42.285 & 41.784 & 41.551 & 41.618 & 27.889 & 27.630 & 27.553 & 27.496 & 19.976 & 19.933 & 19.917 & 19.910 & 1667.171 & 1666.993 & 1667.124 & 1667.158 \\
\hline & 9000 & 67.998 & 67.614 & 67.467 & 67.482 & 43.038 & 42.582 & 42.492 & 42.430 & 28.397 & 28.066 & 27.939 & 27.978 & 21.872 & 21.490 & 21.574 & 21.573 & 1669.207 & 1669.229 & 1669.017 & 1669.082 \\
\hline & 12000 & 68.340 & 67.948 & 67.844 & 67.790 & 43.645 & 43.104 & 43.062 & 43.055 & 29.526 & 29.162 & 29.075 & 29.063 & 23.233 & 23.210 & 23.229 & 23.021 & 1670.454 & 1670.362 & 1670.198 & 1670.487 \\
\hline \multirow[t]{5}{*}{ TS } & 1200 & 60.265 & 60.183 & 60.115 & 60.193 & 32.217 & 32.151 & 32.168 & 32.149 & 18.503 & 18.537 & 18.528 & 18.537 & 13.490 & 13.578 & 13.217 & 13.469 & 1636.800 & \begin{tabular}{|l|}
1636.055 \\
\end{tabular} & 1636.392 & 1636.426 \\
\hline & 3000 & 62.245 & 62.365 & 62.213 & 62.273 & 35.168 & 35.219 & 35.153 & 35.154 & 87 & 21.459 & 21.283 & 21.305 & 17.035 & 16.904 & 16.990 & 16.921 & & 2.123 & 610 & 1652.826 \\
\hline & 6000 & 63.770 & 63.690 & 63.727 & 63.708 & 37.205 & 37.125 & 37.131 & 37.252 & 23.180 & 23.288 & 23.174 & 23.209 & 18.344 & 18.347 & 18.371 & 18.348 & 1660.527 & 1660.413 & 1660.311 & 1660.419 \\
\hline & 9000 & 64.481 & 64.473 & 64.564 & 64.546 & 38.274 & 38.352 & 38.126 & 38.254 & 4.129 & 24.000 & 24.275 & 24.110 & 18.830 & 18.852 & 18.868 & 18.871 & 1663.741 & 1664.157 & 1663.850 & 1663.749 \\
\hline & 12000 & 65.027 & 65.013 & 65.054 & 65.026 & 39.143 & 39.101 & 39.105 & 39.043 & 25.207 & 25.186 & 25.370 & 25.161 & 19.257 & 19.194 & 19.223 & 19.232 & 1666.039 & 666.021 & 1666.035 & 1666.191 \\
\hline \multirow[t]{5}{*}{ SA } & 1200 & \begin{tabular}{|l|}
62.523 \\
\end{tabular} & 62.610 & \begin{tabular}{|l|}
62.322 \\
\end{tabular} & \begin{tabular}{|l|}
62.088 \\
\end{tabular} & 36.643 & 36.493 & 36.154 & 35.933 & 22.105 & 21.875 & 21.890 & 21.650 & 13.051 & 12.865 & 12.857 & 12.782 & 1669.539 & \begin{tabular}{|l|}
1668.425 \\
\end{tabular} & 1666.993 & 1669.549 \\
\hline & 3000 & 64.930 & 64.937 & 64.810 & 64.634 & 40.244 & 40.158 & 40.034 & 39.757 & 5.947 & 25.917 & 25.698 & 25.610 & 17.069 & 17.074 & 17.122 & 16.818 & 1674.405 & 1673.263 & 1673.662 & 1674.654 \\
\hline & 6000 & 66.615 & 66.581 & 66.627 & 66.554 & 42.911 & 42.938 & 42.775 & 42.768 & 28.652 & 28.683 & 28.343 & 28.352 & 18.615 & 18.584 & 18.684 & 18.539 & 1677.910 & 1676.437 & 1677.128 & 1678.012 \\
\hline & 9000 & 67.532 & 67.480 & 67.480 & 67.423 & 44.438 & 44.394 & 44.227 & 44.257 & 30.340 & 30.245 & 30.233 & 30.081 & 19.247 & 19.240 & 19.273 & 19.219 & 1680.058 & 1678.683 & 1678.785 & 1679.600 \\
\hline & 12000 & 68.189 & 68.087 & 68.016 & 67.969 & 45.469 & 45.418 & 45.232 & 45.269 & 31.251 & 31.259 & 31.109 & 31.153 & 19.641 & 19.587 & 19.591 & 19.617 & 1681.037 & 1679.657 & 1679.558 & 1680.794 \\
\hline \multirow{3}{*}{ GA } & 6000 & 99.262 & 98.264 & 94.202 & 86.971 & 98.416 & 94.917 & 75.969 & 51.863 & 164 & 60. & 35.031 & & 12.000 & 11.936 & & & & 720 & & 1676.947 \\
\hline & 9000 & 99.511 & 98.844 & 96.142 & 91.297 & 98.960 & 96.482 & 79.285 & 53.469 & 82.451 & 62.888 & 37.404 & 42.410 & 12.000 & 11.947 & 11.790 & 11.973 & 1677.934 & 1669.617 & 1656.146 & 1677.686 \\
\hline & 12000 & 99.631 & 99.132 & 97.098 & 93.479 & 99.216 & 97.615 & 81.024 & 54.213 & 82.912 & 64.791 & 38.573 & 42.848 & 12.000 & 11.970 & 11.851 & 11.982 & 1678.553 & 1669.102 & 1657.887 & 1678.778 \\
\hline \multirow[t]{5}{*}{ EE } & 1200 & \begin{tabular}{|l|}
95.793 \\
\end{tabular} & & \begin{tabular}{|l|}
70.493 \\
\end{tabular} & 65 & & 75.6 & & & & & & & 11.887 & 11.274 & 110 & & & 649.219 & & 1662.719 \\
\hline & 3000 & 98.399 & 95.677 & 84.508 & 67.933 & 96.647 & 89.959 & 65.792 & 48.535 & 76.919 & 57.889 & 34.027 & 38.150 & 11.976 & 11.859 & 11.566 & 11.860 & 1671.422 & 1660.911 & 1642.799 & 1672.319 \\
\hline & 6000 & 99.201 & 97.833 & 92.211 & 82.942 & 98.342 & 94.761 & 78.203 & 54.035 & 81.406 & 64.034 & 38.173 & & 11.992 & 11.943 & 11.774 & & 6.044 & 298 & & 1675.258 \\
\hline & 9000 & 99.468 & 98.549 & 94.830 & 88.621 & 98.891 & 96.589 & 83.001 & 58.020 & 83.211 & 66.209 & 40.996 & 42. & 11.990 & 11.959 & 11.878 & 11 & 61 & 898 & 165 & 1677.548 \\
\hline & 12000 & 99.603 & 98.917 & 96.116 & 91.477 & 99.166 & 97.449 & 85.251 & 61.961 & 83.910 & 69.133 & 42.627 & 42.432 & 12.000 & 11.972 & 11.912 & 11.984 & 1678.946 & 1669.402 & 1658.208 & 1678.212 \\
\hline \multirow[t]{5}{*}{ EDA } & 1200 & 51.879 & 50.043 & 50.247 & 50.874 & 20.987 & 18.256 & 18.011 & & & 18.995 & 16.654 & & 9.556 & 9.111 & 8.77 & 9.5 & & & & \\
\hline & 3000 & 51.654 & 50.213 & 50.749 & 55.845 & 18.587 & 18.558 & 18.841 & 18.446 & 15.655 & 15.699 & 15.325 & 15.967 & 8.997 & 9.223 & 9.568 & 9.668 & 1672.846 & 1661.352 & 1641.698 & 1672.438 \\
\hline & 6000 & 51.652 & 50.878 & 50.625 & 50.451 & 20.211 & 18.659 & 19.654 & 18.965 & 11.558 & 15.694 & 16.875 & 15.874 & 9.351 & 9.662 & 8.231 & 9.8 & & 1666.444 & 2.521 & 1676.018 \\
\hline & 9000 & 51. & 51. & 50.894 & 52 & 20.111 & 18 & 18.96 & & & 13.325 & 16.895 & 16. & 9.622 & 9.004 & 9.002 & 9.6 & & 1668.926 & 1656 & 1677.307 \\
\hline & 12000 & 51.623 & 51.841 & 50.254 & 50.647 & 20.249 & 20.654 & 18.122 & 18.998 & 17.654 & 16.215 & 16.888 & 17.995 & 9.335 & 9.664 & 8.399 & 9.645 & 1678.552 & 1669.649 & 1658.087 & 1678.341 \\
\hline
\end{tabular}

Table A.3. Offline performance obtained by AHMA and SORIGA for each problem, severity and frequency of change.

\begin{tabular}{|c|c|c|c|c|c|c|c|c|c|c|c|c|c|c|c|c|c|c|c|c|c|}
\hline & & \multirow{2}{*}{\multicolumn{4}{|c|}{$\begin{array}{l}\text { OneMax } \\
\text { severity }\end{array}$}} & \multirow{2}{*}{\multicolumn{4}{|c|}{$\begin{array}{l}\text { Plateau } \\
\text { severity }\end{array}$}} & \multirow{2}{*}{\multicolumn{4}{|c|}{$\begin{array}{c}\text { RoyalRoad } \\
\text { severity }\end{array}$}} & \multirow{2}{*}{\multicolumn{4}{|c|}{$\begin{array}{l}\text { Deceptive } \\
\text { severity }\end{array}$}} & \multicolumn{4}{|c|}{ Knapsack } \\
\hline & & & & & & & & & & & & & & & & & & & $\mathrm{sev}$ & & \\
\hline & change & 0.1 & 0.2 & 0.5 & 0.9 & 0.1 & 0.2 & 0.5 & 0.9 & 0.1 & 0.2 & 0.5 & 0.9 & 0.1 & 0.2 & 0.5 & 0.9 & 0.1 & 0.2 & 0.5 & 0.9 \\
\hline \multirow[t]{5}{*}{ AHMA } & 1200 & \begin{tabular}{|l|}
97.648 \\
\end{tabular} & 95.355 & 90.662 & 90.607 & 95.073 & 89.423 & 76.900 & 77.154 & 79.723 & 62.762 & 45.140 & 42.663 & 65.123 & 57.088 & 53.930 & 65.736 & 679.172 & 1671.414 & 1663.916 & 1669.617 \\
\hline & & 99.060 & .126 & 96.236 & .179 & 98.037 & \begin{tabular}{|l|l|}
95.763 \\
\end{tabular} & 90.229 & 90.253 & 9.845 & \begin{tabular}{|l|}
78.694 \\
\end{tabular} & 62.560 & 61.201 & 3.112 & 64.655 & 56.092 & 75.094 & 1693.010 & 1681.298 & 1667.938 & 1677.169 \\
\hline & 6000 & 531 & 9.069 & 98.119 & .097 & 99.023 & \begin{tabular}{|l|l|}
97.897 \\
\end{tabular} & 95.118 & 95.180 & .771 & 86.590 & 76.134 & 75.379 & 78.593 & 70.534 & 62.542 & 80.269 & 1701.750 & 1689.347 & 671.447 & 1682.675 \\
\hline & 9000 & 688 & 99.379 & 8.750 & 3.727 & 99.350 & \begin{tabular}{|l|}
98.595 \\
\end{tabular} & 96.739 & 96.780 & 19 & \begin{tabular}{|l|}
89.807 \\
\end{tabular} & 82.400 & 82.514 & 81.574 & 74.068 & 65.763 & 82 & 39 & 48 & 89 & 1688.286 \\
\hline & 12000 & 1763 & 99.532 & 99.060 & 99.049 & 99.512 & 98.943 & 97.580 & 97.581 & .731 & .983 & 85.730 & 85.788 & 3.433 & 76.954 & 7.740 & 83.265 & 707.703 & 697.228 & 1676 & 1692.966 \\
\hline \multirow[t]{5}{*}{ SORIGA } & 1200 & 90.016 & 1.473 & 7.956 & 78.296 & 81.042 & 65.262 & 42.366 & 59.845 & .767 & 2.152 & 26.158 & 38.683 & $=$ & 35.562 & 34.111 & 36.985 & 00 & 300 & 00 & 1664.600 \\
\hline & 30 & & 55 & 77 & & 2 & 35 & & 79. & 7.991 & & & & 3 & 4 & & & & & & 665.920 \\
\hline & 60 & 259 & 91.274 & 78.305 & 90.896 & 92.677 & 82.238 & 57.556 & 82.7 & 5.276 & 69.339 & 40.614 & 73.028 & 39.665 & 35.925 & 34.322 & 36 & 40 & 332. & 20 & 332.960 \\
\hline & 9000 & 283 & 93.250 & 82.096 & 92.090 & 94.657 & 86.391 & 61.5 & 83. & 8.848 & $\begin{array}{l}74.916 \\
\end{array}$ & 44.886 & 75. & 40.364 & 36.345 & 34.672 & & 60 & 221 & 221 & 221.947 \\
\hline & 12000 & 97.914 & 94.420 & 84.204 & 93.044 & 95.945 & 88.850 & 64.558 & 84.777 & 90.973 & 77.586 & 47.633 & 75.622 & 41.358 & 37.051 & 34.959 & 36.957 & 166.450 & 166.490 & 166.460 & 166.480 \\
\hline
\end{tabular}

